\title{
Novel Adaptive Sliding Mode Control with Nonlinear Disturbance Observer for SMT Assembly Machine
}

\author{
Rongrong Qian, ${ }^{1,2}$ Minzhou Luo, ${ }^{2}$ Yao Zhao, ${ }^{3}$ and Jianghai Zhao ${ }^{2}$ \\ ${ }^{1}$ School of Information Science and Technology, University of Science and Technology of China, Hefei 230036, China \\ ${ }^{2}$ Institute of Advanced Manufacturing Technology, Hefei Institutes of Physical Science, Chinese Academy of Sciences, \\ Changzhou 213164, China \\ ${ }^{3}$ School of Electrical Engineering, Shanghai University of Electric Power, Shanghai 200090, China
}

Correspondence should be addressed to Yao Zhao; nihaozhaoyao@163.com

Received 15 January 2016; Revised 3 March 2016; Accepted 6 March 2016

Academic Editor: Asier Ibeas

Copyright (C) 2016 Rongrong Qian et al. This is an open access article distributed under the Creative Commons Attribution License, which permits unrestricted use, distribution, and reproduction in any medium, provided the original work is properly cited.

This paper presents a novel adaptive sliding mode control based on nonlinear sliding surface with disturbance observer (ANSMCDOB) for precision trajectory tracking control of a surface mount technology (SMT) assembly machine. A two-degree-of-freedom model with time-varying parameter uncertainties and disturbances is built to describe the first axial mode of the pick-place actuation axis of the machine. According to the principle of variable damping ratio coefficient which makes the system have a nonovershoot transient response and a short settling time in the second-order system, the nonlinear sliding surface is designed for the sliding mode control (SMC). Since the upper bound value of the disturbances is unknown, the adaptive gain estimation is applied to replace the switching gain in the SMC. In order to settle the problem of SMC unrobust to the mismatched parameter uncertainties and disturbances, the nonlinear disturbance observer is introduced to estimate the mismatched disturbances and form the novel controller of ANSMC-DOB. The stability of sliding surfaces and control laws are verified by the Lyapunov functions. The simulation research and comparative experiments are conducted to verify the improvement of positioning accuracy and robustness by the proposed ANSMC-DOB in the SMT assembly machine.

\section{Introduction}

Surface mount technology (SMT) assembly machine, often called chip mounter, is an essential part in the printed circuit boards (PCB) assembly lines. It is mostly composed of $X-Y$ gantry platform. The fast and precision motion is required for the actuation part in the machine. However, the high speed may induce severe mechanical residual vibration which results in long response time and low position accuracy with overshoots [1]. The important thing to deal with this problem is to generate a motion profile firstly and then design a controller to make the actuator following it [2-4]. Apart from the motion profile generation, high performance control algorithms are crucial for the suppression of residual vibrations in the fast and precision trajectory tracking system. Traditional linear control laws such as proportionalintegral-derivative (PID) or proportional-derivative (PD) control are often unable to satisfy these high performance requirements $[4,5]$. Instead, a large number of vibration suppression methods emerge. A pole-placement technique is used in [6] to achieve active vibration damping and high bandwidth disturbance rejection and positioning in the ball screw drive machines. Adaptive feed-forward cancellation with proportional-integral (PI) control is designed in [7] to improve the tracking accuracy at the selected frequency modes. Besides, a variety of tracking control strategies are also proposed [8-11].

Since the parameter perturbation and external disturbance always exist in the industrial machines, the control system includes not only the suppression of the residual vibration, but also the robustness to the disturbance. Sliding mode control (SMC) is employed frequently in the disturbance control system due to its robustness to the parameter perturbation and external disturbance [12-15]. For example, an adaptive robust controller (ARC) is designed in [16-18] to compensate for the effect of unknown model parameters 
and bounded disturbances effectively and provide an online adaptation-based dynamic model identification method.

Another significant key issue in the control system design is to guarantee the transient response and steady-state output error. A prescribed performance control has been presented in $[19,20]$ to keep the maximum overshoot, the convergence rate, and steady-state error in the prespecified values. It is realized by the incorporation of time inequality constraints in the control objective via the introduction of a transformed error [21]. Combining with the variable structure control, another method to improve the system convergence accuracy and transient response speed is presented by Bijnan et al. which is the nonlinear sliding surface [22] used in SMC. It is designed according to the principle of composite nonlinear feedback principle [23]. With this nonlinear sliding surface, the system can have a low damping ratio with fast response initially. As the system gets close to the desired trajectory, the damping ratio is getting bigger to avoid the overshoot. Apparently, it allows a closed-loop system to simultaneously achieve low overshoot and a small settling time, thus resulting in a small error compared to conventional SMC.

However, the SMC with nonlinear sliding surface still has similar weakness to the conventional SMC which is the unrobustness to the mismatched uncertainties and disturbances [24]. This leads to many researches on the SMC design for uncertain systems with mismatched uncertainties. The linear matrix inequality (LMI) based sliding surface design for SMC has been proposed to guarantee the asymptotic stability of full-order system with mismatched uncertainties [25-27]. An adaptive radial basis function neural network is proposed in [28] to compensate for the effects of mismatched uncertainty in the framework of integral SMC. In [29], the robust sliding hyperplane is constructed from a Riccati inequality associated with quadratic stability. All the methods mentioned above are integrated into the SMC to counteract the mismatched disturbance. The nonlinear disturbance observer (NDO) [24, 30-32] can also be used for estimating the mismatched disturbance to design the new SMC with the property of robustness to the mismatched disturbance. A disturbance observer based on the variable structure system theory for minimum-phase dynamical systems with arbitrary relatives has been proposed in [33] when the upper and lower bounds of disturbance are assumed to be known as prior information. However, the prior knowledge of disturbance is always hard to acquire. In order to deal with this problem, a new NDO based on the tracking differentiator is proposed for uncertain dynamic system to estimate many types of uncertain disturbances [34].

In this paper, we propose an application for trajectory tracking control of SMT assembly machine with an improved algorithm based on SMC with nonlinear sliding surface to increase the positioning precision. For the research of SMC with nonlinear sliding surface, two preconditions should be satisfied. One is the prior knowledge of the matched parameter perturbation and disturbance, and the other is that the mismatched parameter and disturbance should be hypothesized to be nonexistent. Both preconditions are hard to acquire in the SMT assembly machine. In order to deal with these problems, the improved algorithm named adaptive

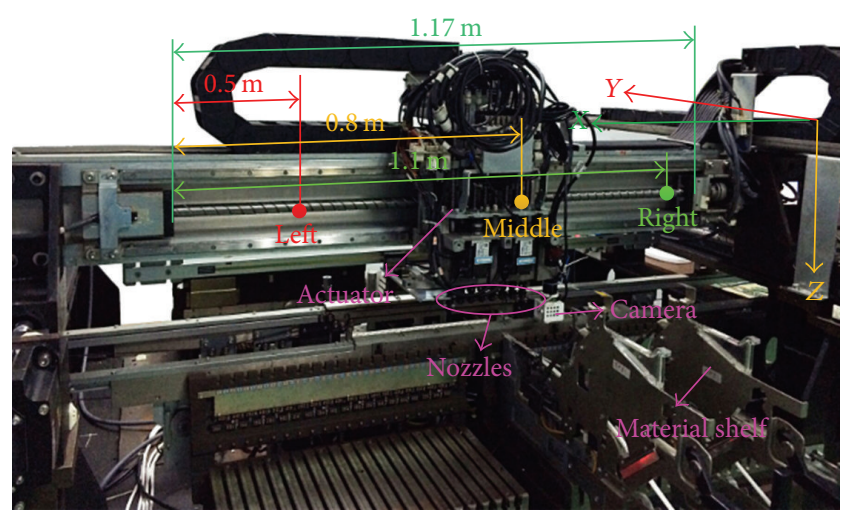

FIGURE 1: SMT assembly machine.

sliding mode control based on nonlinear sliding surface and disturbance observer (ANSMC-DOB) is proposed and applied in the SMT assembly machine. The paper is organized as follows. Firstly, the system prototype and hardware structure of control system for the machine are given. A two-degree-of-freedom (two-DOF) model with matched and mismatched disturbance is induced and identified by the frequency response experiment. Supposing that the two preconditions mentioned above are satisfied, a SMC with nonlinear sliding surface for the two-DOF model is presented. This system has the distinct advantage of good response performance with nonovershoot and a short settling time. However, considering the reality that the prior knowledge for matched parameter perturbation and disturbance is hard to acquire, the adaptive parameter estimation is designed to determine the switching gain. Meanwhile, the mismatched disturbance also exists in reality. Therefore, with the purpose of improving the positioning accuracy and dealing with the mismatched disturbance, the nonlinear disturbance observer is proposed to estimate the mismatched disturbance. A new sliding surface is designed by combining nonlinear sliding surface with nonlinear disturbance observer and the novel controller law of ANSMC-DOB is presented. The stabilities of sliding surfaces and control laws are verified by the Lyapunov function. In addition, controller simulation research and comparative experiments are conducted. The conclusion and outlook are drawn in the end.

\section{Hardware Platform of SMT Assembly Machine}

A three-coordinate prototype of the $X-Y$ gantry platform with actuator has been developed for fast and precision SMT assembly machine. It is essentially composed of three large stroke ball screw drive systems and shown in Figure 1. The pick-place actuator is in the $x$-axis ball screw drive system. The $x$-axis of ball screw drive system is defined as the pickplace actuation axis in this paper. The motion of nozzles in the actuator is in the $z$-axis direction. The motion of $y$ axis direction is the synchronous movement of the pick-place actuation axis on two ball screw drive systems. The length of ball screw in the pick-place actuation axis is $1.17 \mathrm{~m}$. There are 


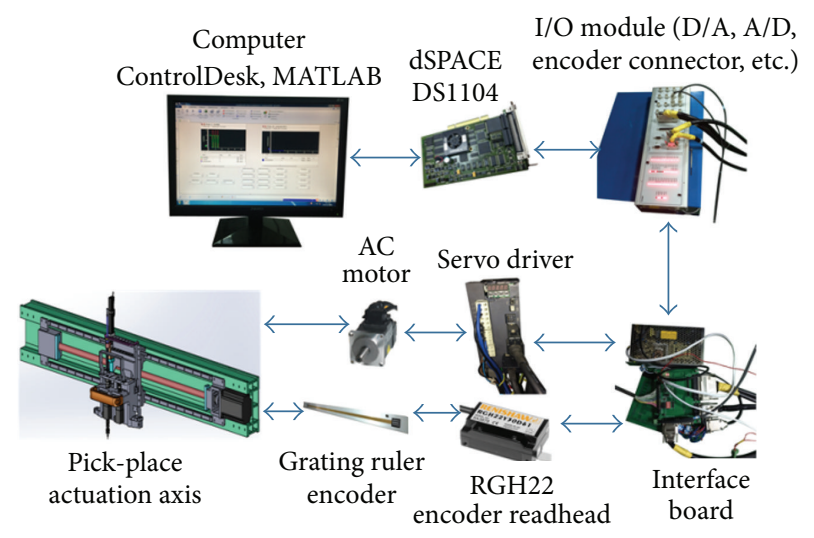

FIgURE 2: Schematic diagram of the control system.

8 nozzles in the actuator for the material suction and placing. In order to describe the control experiment conveniently later, the three initial locations of actuator are marked as left, middle, and right, respectively.

The schematic diagram of control system for the pickplace actuation axis is shown in Figure 2. An AC servo motor is used to drive the ball screw to rotate and actuate the actuator to move axially. The lead of ball screw is $40 \mathrm{~mm} / \mathrm{rev}$. There are two position measuring sensors which are the rotary and linear encoders. The rotary encoder is in the AC servo motor. The linear encoder of high precision grating ruler is fixed in the machine base. The resolution of both encoders has been set as $1 \mu \mathrm{m}$ equivalent in the actuator motion direction. The AC motor is driven by a specific servo driver which is configured in the torque control mode. Since the response of electrical control is faster than mechanical motion and the current loop bandwidth of servo motor is high enough, the current command to the servo driver can be considered as the actual current. The positioning control algorithm is firstly simulated in MATLAB/Simulink and then compiled and implemented in dSPACE DS1104 system by ControlDesk software. The interface board is designed to realize the correct signal connections between DS1104 I/O module and servo driver/grating ruler readhead.

In the assembly line of $\mathrm{PCB}$, fast and precision positioning of the chip mounter is the key research point to acquire high productivity and good quality. For the dimension of chips is gradually decreased, the tracking error of the actuator should be decreased as well. The target of this paper is to design an effective control law to make the positioning error of the actuator in the range of $\pm 10 \mu \mathrm{m}$ with high robust and high transient response performance.

\section{Two-DOF Model of the Pick-Place Actuation Axis}

In the ball screw drive system with large stroke, the first axial vibration mode has the most influence on the linear positioning accuracy and the available control bandwidth [35]. It is mainly caused by the flexible characteristics of the

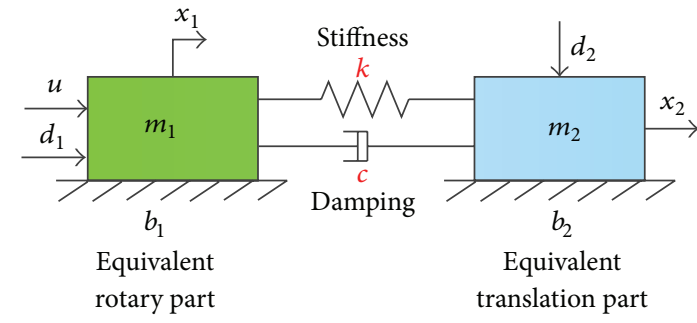

Figure 3: Two-DOF model for pick-place actuation axis.

translating components and the elastic interaction between the worktable, nut, and ball screw. In order to achieve high speed and high precision for the pick-place actuation axis, the first axial vibration mode has to be modeled and controlled well. A two-mass drive system, shown in Figure 3, is used to capture this mode and the two-DOF dynamic model of the system is expressed as

$$
\begin{aligned}
m_{1} \ddot{x}_{1}= & -b_{1} \dot{x}_{1}+k\left(x_{2}-x_{1}\right)+c\left(\dot{x}_{2}-\dot{x}_{1}\right)+u(t) \\
& +d_{1}(t), \\
m_{2} \ddot{x}_{2}= & -b_{2} \dot{x}_{2}+k\left(x_{1}-x_{2}\right)+c\left(\dot{x}_{1}-\dot{x}_{2}\right)+d_{2}(t),
\end{aligned}
$$

where $m_{1}$ is the rotary inertia equivalent to the motor shaft and $m_{2}$ is the inertia of actuator. $b_{1}$ is the viscous friction between the motor shaft and rotary bearing while $b_{2}$ is the viscous friction of the bilateral guides. $k$ represents the total equivalent axial stiffness. $c$ represents the damping in the preloaded nut. $u(t)$ is the control input voltage used to generate the motor torque to actuate the ball screw drive system. $x_{1}(t)$ is the equivalent rotary displacement of the motor shaft. $x_{2}(t)$ is the equivalent rotary displacement of actuator multiplied by the screw pitch. In the ball screw driven system of SMT assembly machine, the resonant modes, such as axial modes and torsional modes, are excited by the high speed and high acceleration motion. As the actuator moves along the ball screw, the mode frequencies shift due to the distributed stiffness and inertia of flexible ball screw. The first axial mode has a strong shift when the position of actuator is changing [35]. It directly causes the variations of inertia, damping, and stiffness in (1a) and (1b) during the operations, thus leading to the time-varying parametric uncertainties. Meanwhile, during the process of the actuator moving, the 8 nozzles in the actuator are driven by eight small servo motors to rotate and adjust the chip angle according to the circuit board scene captured by the camera. This motion in the actuator influences the trajectory tracking accuracy when the actuator is moving and could be considered as the load disturbance. The friction in the SMT assembly machine also changes with the variation of the actuator's moving velocity. Therefore, for the sake of analysis, $d_{1}(t)$ and $d_{2}(t)$ are used here to represent the integration of the time-varying parameter uncertainties and load disturbance mentioned above (see Figure 3 ).

In this paper, $d_{1}(t)$ is regarded as the matched parameter uncertainties and disturbance to the input and $d_{2}(t)$ is defined as the mismatched disturbance. In order to realize 
the controller design, the following assumption about the performance of $d_{1}(t)$ and $d_{2}(t)$ is given.

Assumption 1. The parameter uncertainties and equivalent external disturbance $d_{1}(t)$ and $d_{2}(t)$ are bounded and they can be described as

$$
\begin{aligned}
& \left|d_{1}(t)\right|<q_{1}, \\
& \left|d_{2}(t)\right|<q_{2},
\end{aligned}
$$

where $q_{1}, q_{2}>0$.

For most of the mechanical systems with finite load, this assumption can be available. From (1a) and (1b), the transfer functions of two-DOF model are acquired as follows:

$$
\begin{aligned}
& G_{1}(s)=\frac{x_{1}(s)}{u(s)}=\frac{m_{2} s^{2}+\left(b_{2}+c\right) s+k}{a_{4} s^{4}+a_{3} s^{3}+a_{2} s^{2}+a_{1} s}, \\
& G_{2}(s)=\frac{x_{2}(s)}{u(s)}=\frac{c s+k}{a_{4} s^{4}+a_{3} s^{3}+a_{2} s^{2}+a_{1} s},
\end{aligned}
$$

where $a_{4}=m_{1} m_{2}, a_{3}=m_{1}\left(b_{2}+c\right)+m_{2}\left(b_{1}+c\right), a_{2}=k\left(m_{1}+\right.$ $\left.m_{2}\right)+b_{1} b_{2}+b_{1} c+b_{2} c$, and $a_{1}=k\left(b_{1}+b_{2}\right)$.

In order to identify the accurate model of (1a) and (1b), the frequency response experiment is conducted by exciting the AC motor with a series of sinusoidal torque voltage signals. The solid line in Figure 4 is the first axial mode frequency response. The pole-placement principle through the transfer functions ( $3 a$ ) and ( $3 b$ ) is used for identifying the parameters of (1a) and (lb). The identified result is

$$
\begin{aligned}
m_{1} & =5.4741 \times 10^{-4}\left(\mathrm{~V} / \mathrm{rad} / \mathrm{s}^{2}\right), \\
m_{2} & =10.0841 \times 10^{-4}\left(\mathrm{~V} / \mathrm{rad} / \mathrm{s}^{2}\right), \\
k & =353.2717(\mathrm{~V} / \mathrm{rad}), \\
c & =0.020(\mathrm{~V} / \mathrm{rad} / \mathrm{s}), \\
b_{1} & =0.0013(\mathrm{~V} / \mathrm{rad} / \mathrm{s}), \\
b_{2} & =0.0013(\mathrm{~V} / \mathrm{rad} / \mathrm{s}) .
\end{aligned}
$$

The dashed line in Figure 4 is the frequency response simulation of (1a) and (1b) with the identified parameters above. It can be seen that the frequency response of the model is in good agreement with the measurement result of first axial vibration mode. The state space model which is
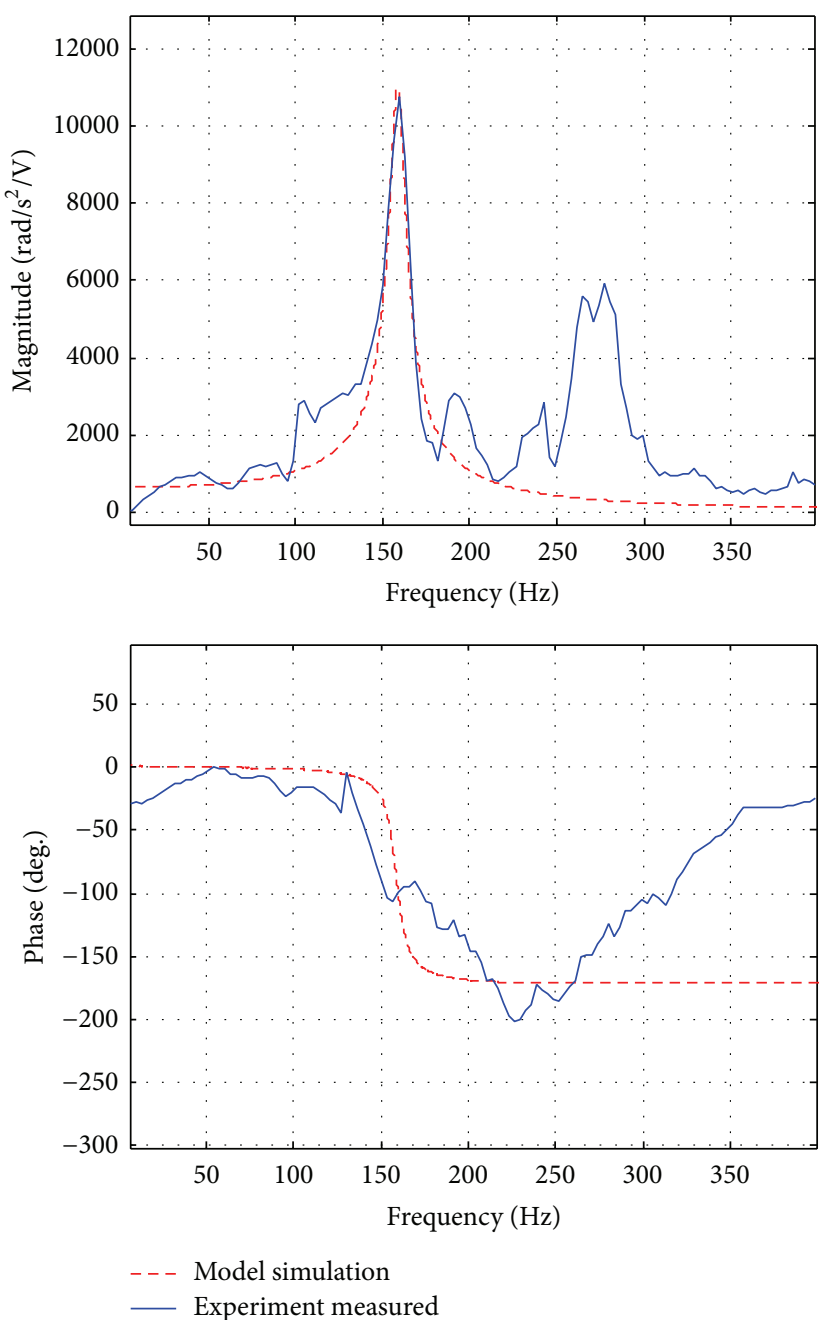

FIGURE 4: Simulated and measured frequency response.

transferred from (1a) and (1b) with the state vector $\mathbf{x}(t)=$ $\left[x_{2}(t), x_{1}(t), \dot{x}_{2}(t), \dot{x}_{1}(t)\right]^{\mathrm{T}}$ is inferred as follows:

$$
\begin{aligned}
& {\left[\begin{array}{l}
\dot{x}_{2}(t) \\
\dot{x}_{1}(t) \\
\ddot{x}_{2}(t) \\
\ddot{x}_{1}(t)
\end{array}\right]} \\
& =\left[\begin{array}{cccc}
0 & 0 & 1 & 0 \\
0 & 0 & 0 & 1 \\
-\frac{k}{m_{2}} & \frac{k}{m_{2}} & -\frac{b_{2}+c}{m_{2}} & \frac{c}{m_{2}} \\
\frac{k}{m_{1}} & -\frac{k}{m_{1}} & \frac{c}{m_{1}} & -\frac{b_{1}+c}{m_{1}}
\end{array}\right]\left[\begin{array}{c}
x_{2}(t) \\
x_{1}(t) \\
\dot{x}_{2}(t) \\
\dot{x}_{1}(t)
\end{array}\right] \\
& +\left[\begin{array}{c}
0 \\
0 \\
0 \\
\frac{1}{m_{1}}
\end{array}\right] u(t)+\left[\begin{array}{c}
0 \\
0 \\
0 \\
\frac{1}{m_{1}}
\end{array}\right] d_{1}(t)+\left[\begin{array}{c}
0 \\
\frac{1}{m_{2}} \\
0
\end{array}\right] d_{2}(t) .
\end{aligned}
$$


The state vector is divided into two parts as $\mathbf{z}_{1}(t)=$ $\left[x_{2}(t), x_{1}(t), \dot{x}_{2}(t)\right]^{\mathrm{T}}$ and $z_{2}(t)=\dot{x}_{1}(t)$. The corresponding state space model is

$$
\begin{aligned}
{\left[\begin{array}{c}
\dot{\mathbf{z}}_{1}(t) \\
\dot{z}_{2}(t)
\end{array}\right]=} & {\left[\begin{array}{ll}
\mathbf{A}_{11} & \mathbf{A}_{12} \\
\mathbf{A}_{21} & A_{22}
\end{array}\right]\left[\begin{array}{l}
\mathbf{z}_{1}(t) \\
z_{2}(t)
\end{array}\right]+\left[\begin{array}{c}
\mathbf{0}_{3 \times 1} \\
B_{1}
\end{array}\right] u(t) } \\
& +\left[\begin{array}{c}
\mathbf{0}_{3 \times 1} \\
B_{1}
\end{array}\right] d_{1}(t)+\left[\begin{array}{c}
\mathbf{B}_{2} \\
0
\end{array}\right] d_{2}(t),
\end{aligned}
$$

where

$$
\begin{aligned}
& \mathbf{A}_{11}=\left[\begin{array}{ccc}
0 & 0 & 1 \\
0 & 0 & 0 \\
-\frac{k}{m_{2}} & \frac{k}{m_{2}} & -b_{2}-c
\end{array}\right] \text {, } \\
& \mathbf{A}_{12}=\left[\begin{array}{c}
0 \\
1 \\
\frac{c}{m_{2}}
\end{array}\right], \\
& \mathbf{A}_{21}=\left[\begin{array}{lll}
\frac{k}{m_{1}} & -\frac{k}{m_{1}} & \frac{c}{m_{1}}
\end{array}\right] \text {, } \\
& A_{22}=-\frac{b_{1}+c}{m_{1}}, \\
& B_{1}=\frac{1}{m_{1}} \text {, } \\
& \mathbf{B}_{2}=\left[\begin{array}{c}
0 \\
0 \\
\frac{1}{m_{2}}
\end{array}\right], \\
& \mathbf{A}=\left[\begin{array}{ll}
\mathbf{A}_{11} & \mathbf{A}_{12} \\
\mathbf{A}_{21} & A_{22}
\end{array}\right] \text {. }
\end{aligned}
$$

\section{ANSMC-DOB for SMT Assembly Machine}

In order to introduce the proposed control algorithm, a comparative step response of second-order systems with different damping ratios is shown in Figure 5. The secondorder system is given as

$$
G(s)=\frac{25}{s^{2}+10 \xi \cdot s+25}
$$

The damping ratios $\xi$ of four systems are given as

Sys.ud (underdamped system): $\xi=0.4$,

Sys.cd (critical damped system): $\xi=1$,

Sys.od (overdamped system): $\xi=1.4$,

Sys.vd (variable damped system): $\xi=14.2 e^{-5\left((y-r)^{2}\right)}$,

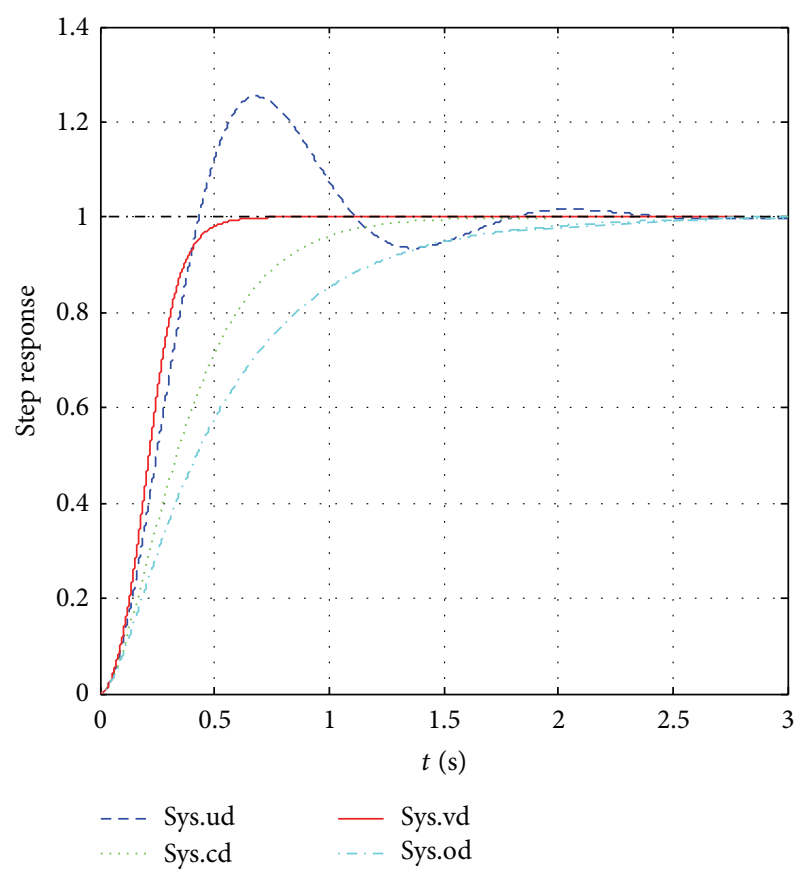

FIGURE 5: Comparative step responses of second-order system with different damping ratios.

where $y$ and $r$ represent the system actual output and desired output, respectively. It can be concluded from Figure 5 that the system with small damping ratio has a fast response but the system overshoot is large. The system with big damping ratio, such as Sys.cd and Sys.od, has a nonovershoot response but the response speed is slow. The system Sys.vd combines the advantages of the previous two kinds of systems and has the best transient response performance with nonovershoot and short settling time. The variable damping ratio in Sys.vd is changing from a tiny value to a high value as the system actual output is gradually moving close to the expected one. Spurred by this principle, the novel ANSMC-DOB is presented in this section for two-DOF model of the pick-place actuation axis.

4.1. Nonlinear Sliding Surface. The novel nonlinear sliding surface is firstly proposed by Bijnan et al. [22] for the system only with matched disturbance to realize the response with short settling time and nonovershoot. Assume that the mismatched disturbance $d_{2}(t)$ in $(6)$ is neglected here and the desired state trajectory is defined as

$$
\begin{aligned}
\mathbf{z}_{r}(t) & =\left[\begin{array}{lll}
\mathbf{z}_{1 r}(t) & z_{2 r}(t)
\end{array}\right]^{\mathrm{T}}, \\
\mathbf{z}_{1 r}(t) & =\left[\begin{array}{lll}
x_{2 r}(t) & x_{1 r}(t) & \dot{x}_{2 r}(t)
\end{array}\right]^{\mathrm{T}}, \\
z_{2 r}(t) & =\dot{x}_{1 r}(t) .
\end{aligned}
$$

The state errors are illustrated as

$$
\begin{aligned}
& \mathbf{e}_{1}(t)=\mathbf{z}_{1 r}(t)-\mathbf{z}_{1}(t), \\
& e_{2}(t)=z_{2 r}(t)-z_{2}(t) .
\end{aligned}
$$


The nonlinear sliding surface $s(\mathbf{z}, t)$ for counteracting the matched disturbance $d_{1}(t)$ is defined as

$$
\begin{aligned}
s(\mathbf{z}, t) & =\boldsymbol{\sigma}(y, r) \cdot \mathbf{e}(t) \\
& =\left[\mathbf{F}-\varphi(y, r) \mathbf{A}_{12}^{\mathrm{T}} \mathbf{P} \quad 1\right]\left[\begin{array}{l}
\mathbf{e}_{1}(t) \\
e_{2}(t)
\end{array}\right],
\end{aligned}
$$

where $\boldsymbol{\sigma}(y, r)=\left[\mathbf{F}-\varphi(y, r) \mathbf{A}_{12}^{\mathrm{T}} \mathbf{P} 1\right] . y$ and $r$ are the actual and desired actuator equivalent rotary displacement. $\mathbf{F}$ is chosen to satisfy the condition that $\mathbf{A}_{11}-\mathbf{A}_{12} \mathbf{F}$ has stable eigenvalues and its dominant poles have a very low damping ratio [22]. $\mathbf{P}$ is the positive definite symmetric matrix which is chosen based on the final damping ratio and the Lyapunov equation below:

$$
\left(\mathbf{A}_{11}-\mathbf{A}_{12} \mathbf{F}\right)^{\mathrm{T}} \mathbf{P}+\mathbf{P}\left(\mathbf{A}_{11}-\mathbf{A}_{12} \mathbf{F}\right)=-\mathbf{W},
$$

where $\mathbf{W}$ is a known positive definite matrix. $\varphi(y, r)$ is a negative function and is continuously differentiable with respect to $y$. It has a similar effect to the variable damping ratio in Sys.vd which is to gradually increase the damping ratio of the control system. $\varphi(y, r)$ is chosen as follows:

$$
\varphi(y, r)=-\beta\left(e^{-\rho(y-r)^{2}}\right),
$$

where $\beta$ is used to adjust the damping ratio and satisfies $\beta>0$. $\rho$ should be big enough to make $\varphi(y, r)$ be a tiny value initially. According to the control principle of the SMC, the system will be forced to the sliding surface (11) by the control law and then the following equation is satisfied:

$$
s(\mathbf{z}, t)=0 .
$$

Combining (6), (10a), (10b), and (14), it yields that

$$
\begin{aligned}
& e_{2}(t)=-\mathbf{F e}_{1}(t)+\varphi(y, r) \mathbf{A}_{12}^{\mathrm{T}} \mathbf{P e}_{1}(t), \\
& \dot{\mathbf{e}}_{1}(t)=\left(\mathbf{A}_{11}-\mathbf{A}_{12} \mathbf{F}+\mathbf{A}_{12} \varphi(y, r) \mathbf{A}_{12}^{\mathrm{T}} \mathbf{P}\right) \mathbf{e}_{1}(t) .
\end{aligned}
$$

It can be observed from (15) that

$$
\mathbf{e}_{1}(t)=\mathbf{0} \Longrightarrow e_{2}(t)=0 .
$$

In order to analyze the convergence performance of the system state errors, the Lyapunov function for subsystem (16) is defined as

$$
V_{1}(t)=\mathbf{e}_{1}^{\mathrm{T}}(t) \mathbf{P e}_{1}(t) .
$$

Then, it follows that

$$
\begin{aligned}
\dot{V}_{1}(t)= & \dot{\mathbf{e}}_{1}^{\mathrm{T}}(t) \mathbf{P} \mathbf{e}_{1}(t)+\mathbf{e}_{1}^{\mathrm{T}}(t) \mathbf{P} \dot{\mathbf{e}}_{1}(t) \\
= & \mathbf{e}_{1}^{\mathrm{T}}(t)\left(\mathbf{A}_{11}-\mathbf{A}_{12} \mathbf{F}\right)^{\mathrm{T}} \mathbf{P} \mathbf{e}_{1}(t) \\
& +\mathbf{e}_{1}^{\mathrm{T}}(t) \mathbf{P}\left(\mathbf{A}_{11}-\mathbf{A}_{12} \mathbf{F}\right) \mathbf{e}_{1}(t) \\
& +2 \mathbf{e}_{1}^{\mathrm{T}}(t) \mathbf{P} \mathbf{A}_{12} \varphi(y, r) \mathbf{A}_{12}^{\mathrm{T}} \mathbf{P} \mathbf{e}_{1}(t)<0 .
\end{aligned}
$$

Therefore, the error $\mathbf{e}_{1}(t)$ is asymptotic convergence to zero as well as $e_{2}(t)$.
4.2. ANSMC. The SMC based on nonlinear sliding surface is designed to force the system state errors (10a) and (10b) to move to the predetermined sliding surface (11) and keep on it afterwards. The exponential approaching law (20) which has the advantages of low chattering in the case of switching control is used here to design the controller:

$$
\dot{s}(\mathbf{z}, t)=-k_{1} s(\mathbf{z}, t)-k_{2} \operatorname{sgn}(s(\mathbf{z}, t)),
$$

where $k_{1}>0$ and $k_{2}>q_{1} / m_{1}$.

Since the upper bound of matched disturbance $q_{1}$ is unknown and difficult to acquire, the adaptive parameter estimation $\hat{k}(t)$ as (21) is introduced here instead of the switching gain $k_{2}$. Hence,

$$
\dot{\hat{k}}(t)=\mu|s(\mathbf{z}, t)|,
$$

where $\mu>0$. Taking the derivative of the sliding surface (11) yields that

$$
\begin{aligned}
\dot{s}(\mathbf{z}, t)= & \left.-\frac{\mathrm{d} \varphi(y, r)}{\mathrm{d} t} \mathbf{A}_{12}^{\mathrm{T}} \mathbf{P} \quad 0\right]\left[\begin{array}{l}
\mathbf{e}_{1}(t) \\
e_{2}(t)
\end{array}\right] \\
& +\left[\mathbf{F}-\varphi(y, r) \mathbf{A}_{12}^{\mathrm{T}} \mathbf{P} \quad 1\right]\left[\begin{array}{l}
\dot{\mathbf{e}}_{1}(t) \\
\dot{e}_{2}(t)
\end{array}\right] \\
= & -\frac{\mathrm{d} \varphi(y, r)}{\mathrm{d} t} \mathbf{A}_{12}^{\mathrm{T}} \mathbf{P e}_{1}(t) \\
& +\left(\mathbf{F}-\varphi(y, r) \mathbf{A}_{12}^{\mathrm{T}} \mathbf{P}\right) \dot{\mathbf{e}}_{1}(t)+\dot{e}_{2}(t) \\
= & -\frac{\mathrm{d} \varphi(y, r)}{\mathrm{d} t} \mathbf{A}_{12}^{\mathrm{T}} \mathbf{P} \mathbf{e}_{1}(t) \\
& +\left(\mathbf{F}-\varphi(y, r) \mathbf{A}_{12}^{\mathrm{T}} \mathbf{P}\right)\left(\dot{\mathbf{z}}_{1 r}-\dot{\mathbf{z}}_{1}\right) \\
& +\left(\dot{z}_{2 r}-\dot{z}_{2}\right) \\
= & -\frac{\mathrm{d} \varphi(y, r)}{\mathrm{d} t} \mathbf{A}_{12}^{\mathrm{T}} \mathbf{P} \mathbf{e}_{1}(t)+\boldsymbol{\sigma}(y, r) \dot{\mathbf{z}}_{r}(t) \\
& -\boldsymbol{\sigma}(y, r) \mathbf{A z}(t)-B_{1} d_{1}(t)-B_{1} u(t) .
\end{aligned}
$$

Combining with (20)-(22), the control law and its existence are discussed in the following theorem.

Theorem 2. The control law

$$
\begin{array}{r}
u(t)=B_{1}^{-1}\left(-\frac{\mathrm{d} \varphi(y, r)}{\mathrm{d} t} \mathbf{A}_{12}^{\mathrm{T}} \mathbf{P e}_{1}(t)+\boldsymbol{\sigma}(y, r) \dot{\mathbf{z}}_{r}(t)\right. \\
\left.-\boldsymbol{\sigma}(y, r) \mathbf{A z}(t)+k_{1} s(\mathbf{z}, t)+\widehat{k}(t) \operatorname{sgn}(s(\mathbf{z}, t))\right)
\end{array}
$$

forces the system state error $\mathbf{e}(t)$ to move from any initial condition to the sliding surface (11) in finite time and thereafter to remain on it. In the above control law, the parameter $k_{1}$ should satisfy $k_{1}>0$. 


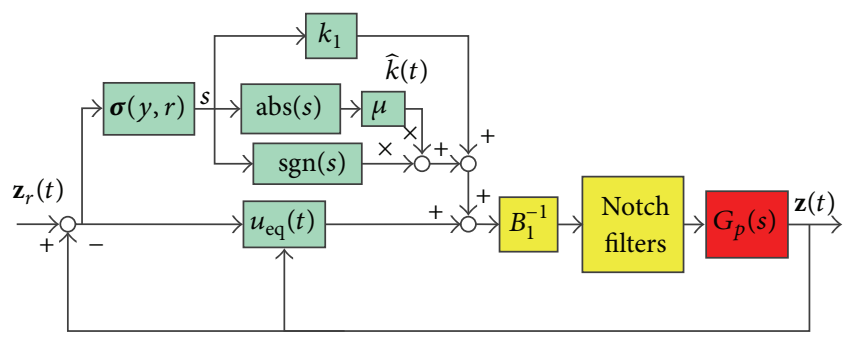

FIGURE 6: The block diagram of ANSMC.

Proof. To verify the stability of ANSMC, consider a quadratic function as

$$
V_{2}(t)=\frac{1}{2} s^{2}(\mathbf{z}, t)+\frac{1}{2 \mu}\left(\widehat{k}(t)-\frac{q_{1}}{m_{1}}\right)^{2} .
$$

Using (21)-(22), it follows that

$$
\begin{aligned}
& \dot{V}_{2}(t)=s(\mathbf{z}, t) \dot{s}(\mathbf{z}, t)+\frac{1}{\mu}\left(\widehat{k}(t)-\frac{q_{1}}{m_{1}}\right) \dot{\hat{k}}(t) \\
& =s(\mathbf{z}, t) \cdot\left(-\frac{\mathrm{d} \varphi(y, r)}{\mathrm{d} t} \mathbf{A}_{12}^{\mathrm{T}} \mathbf{P e}_{1}(t)+\boldsymbol{\sigma}(y, r) \dot{\mathbf{z}}_{r}(t)\right. \\
& \left.-\boldsymbol{\sigma}(y, r) \mathbf{A z}(t)-B_{1} d_{1}(t)-B_{1} u(t)\right)+(\widehat{k}(t) \\
& \left.-\frac{q_{1}}{m_{1}}\right)|s(\mathbf{z}, t)|=s(\mathbf{z}, t) \cdot\left(-k_{1} s(\mathbf{z}, t)\right. \\
& \left.-\widehat{k}(t) \operatorname{sgn}(s(\mathbf{z}, t))-B_{1} d_{1}(t)\right)+\left(\widehat{k}(t)-\frac{q_{1}}{m_{1}}\right) \\
& \cdot|s(\mathbf{z}, t)| \leq-k_{1} s(\mathbf{z}, t)^{2}+\frac{|s(\mathbf{z}, t)|}{m_{1}} \cdot\left(\left|d_{1}(t)\right|-q_{1}\right) .
\end{aligned}
$$

From (1a) and (1b), (25) implies that $\dot{V}_{2}(t)<0$. This completes the proof.

Figure 6 illustrates the corresponding block diagram of ANSMC with the notch filters discussed later. In Figure 6, $u_{\text {eq }}(t)$ is acquired by the equivalent control which is represented as

$$
\begin{aligned}
u_{\mathrm{eq}}(t)= & -\frac{\mathrm{d} \varphi(y, r)}{\mathrm{d} t} \mathbf{A}_{12}^{\mathrm{T}} \mathbf{P e}_{1}(t)+\boldsymbol{\sigma}(y, r) \dot{\mathbf{z}}_{r}(t) \\
& -\boldsymbol{\sigma}(y, r) \mathbf{A z}(t) .
\end{aligned}
$$

4.3. ANSMC-DOB. As previously mentioned, ANSMC has the advantages in the transient response performance and robustness to system with matched disturbance. However, the mismatched disturbance $d_{2}(t)$ cannot be neglected in the system and now it is considered in the controller design in this section.

Firstly, (16) is updated as

$$
\begin{aligned}
\dot{\mathbf{e}}_{1}(t)= & \left(\mathbf{A}_{11}-\mathbf{A}_{12} \mathbf{F}+\mathbf{A}_{12} \varphi(y, r) \mathbf{A}_{12}^{\mathrm{T}} \mathbf{P}\right) \mathbf{e}_{1}(t) \\
& -\mathbf{B}_{2} d_{2}(t) .
\end{aligned}
$$

Equation (27) implies that the actual states cannot be driven to the desired values and even the controller (23) makes the system state errors reach the sliding mode (11). Therefore, the ANSMC based on disturbance observer is presented. The nonlinear disturbance observer proposed in [36] is applied here to estimate $d_{2}(t)$ as follows:

$$
\begin{aligned}
\hat{d}_{2}(t) & =\eta(t)+h \dot{x}_{2}(t), \\
\dot{\eta}(t) & =-h \eta(t)-h^{2} \dot{x}_{2}(t)-h\left(-\frac{k}{m_{2}} x_{2}(t)\right. \\
+ & \left.\frac{k}{m_{2}} x_{1}(t)-\frac{b_{2}+c}{m_{2}} \dot{x}_{2}(t)+\frac{c}{m_{2}} \dot{x}_{1}(t)\right),
\end{aligned}
$$

where $h>0$.

In order to analyze the convergence characteristics of the disturbance observer, the following assumption should be given [37].

Assumption 3. The derivative of $d_{2}(t)$ is bounded and $\dot{d}_{2}(t)$ satisfies the following equation:

$$
\lim _{t \rightarrow \infty} \dot{d}_{2}(t)=0 \text {. }
$$

Under the normal operation, the major disturbance in SMT assembly machine comes from the moving of small servo motors in the actuator and the mechanical friction. Due to the rigid mechanism design of actuator and the continuous motion of servo motors, the disturbance caused by the servo motors' motion can be considered as bounded and continuous. The friction is also bounded and continuous in most mechanical systems. Therefore, the derivative of $d_{2}(t)$ is bounded. In the mechatronics design, the mechanical dynamics are always required to be much slower than the electrical dynamics. Hence, even though there is no prior information about $\dot{d}_{2}(t)$, it is reasonable to suppose that $\dot{d}_{2}(t)=0$ which implies that the disturbance varies slower relative to the disturbance observer dynamics.

The mismatched disturbance estimation error is defined as

$$
e_{d}(t)=d_{2}(t)-\widehat{d}_{2}(t) .
$$

Taking the derivative of (30) and combining with (28a) and (28b) yield

$$
\begin{aligned}
\dot{e}_{d}(t)= & \dot{d}_{2}(t)-\dot{\hat{d}}_{2}(t)=\dot{d}_{2}(t)-\dot{\eta}(t)-h \ddot{x}_{2}(t) \\
= & \dot{d}_{2}(t)+h \eta(t)+h^{2} \dot{x}_{2}(t)+h\left(\ddot{x}_{2}(t)-d_{2}(t)\right) \\
& -h \ddot{x}_{2}(t)=\dot{d}_{2}(t)-h\left(d_{2}(t)-\widehat{d}_{2}(t)\right) .
\end{aligned}
$$

When $t \rightarrow \infty$, (31) has

$$
\dot{e}_{d}(t)+h e_{d}(t)=0 .
$$

From the equation above, we can get a lemma about the convergence performance of the nonlinear disturbance observer. 


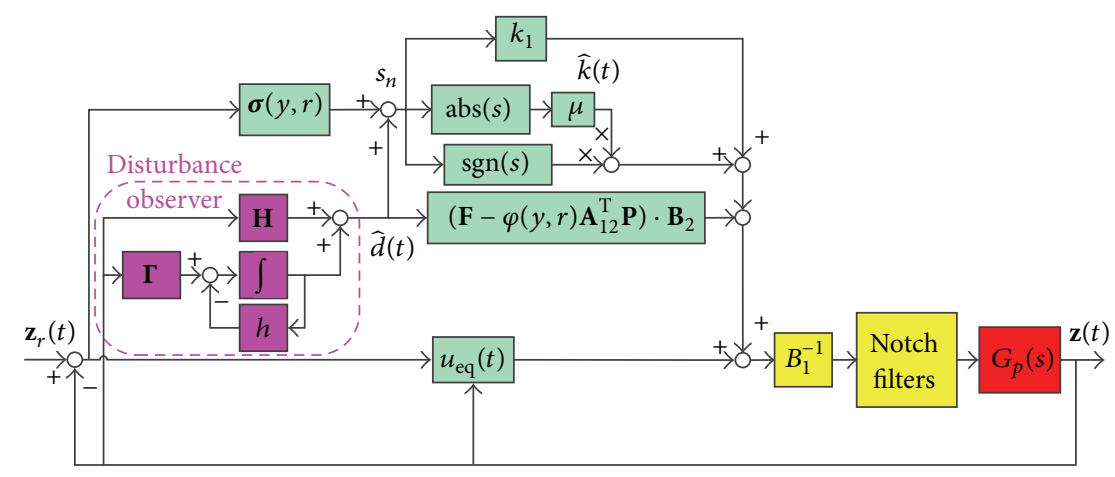

FIgURE 7: The block diagram of ANSMC-DOB.

Lemma 4. For system (6) satisfying Assumptions 1 and 3, when $h$ is chosen as $h>0$, the nonlinear disturbance observer $\widehat{d}_{2}(t)$ of (28a) and (28b) is asymptotically convergent to the actual value of $d_{2}(t)$.

The new sliding surface based on disturbance observer is designed as

$$
\begin{aligned}
s_{n}(\mathbf{z}, t) & =\boldsymbol{\sigma}(y, r) \mathbf{z}(t)+\widehat{d}_{2}(t) \\
& =\left[\mathbf{F}-\varphi(y, r) \mathbf{A}_{12}^{\mathrm{T}} \mathbf{P} \quad 1\right]\left[\begin{array}{l}
\mathbf{e}_{1}(t) \\
e_{2}(t)
\end{array}\right]+\widehat{d}_{2}(t) .
\end{aligned}
$$

According to Lemma 4, the disturbance estimation error is bounded and it follows that

$$
e_{d}^{*}=\sup _{t>0}\left|e_{d}(t)\right| \text {. }
$$

Let $\delta(y, r)=\left(\left(\mathbf{F}-\varphi(y, r) \mathbf{A}_{12}^{\mathrm{T}}\right) \mathbf{P} \cdot \mathbf{B}_{2}-h\right) \cdot e_{d}^{*} ;$ from (34) and Assumption 1 , the upper bound of $|\delta(y, r)|+\left|B_{1} \cdot d_{1}(t)\right|$ exists and can be defined as

$$
|\delta(y, r)|+\left|B_{1} \cdot d_{1}(t)\right|<q_{3} .
$$

For the proposed control law of ANSMC-DOB with sliding surface (33) and its stability performance, a theorem is acquired in the following.

Theorem 5. The control law

$$
\begin{aligned}
& u_{n}(t)=B_{1}^{-1}\left(-\frac{\mathrm{d}}{\mathrm{d} t} \varphi(y, r) \mathbf{A}_{12}^{\mathrm{T}} \mathbf{P e}_{1}(t)+\boldsymbol{\sigma}(y, r) \dot{\mathbf{z}}_{r}(t)\right. \\
& -\boldsymbol{\sigma}(y, r) \mathbf{A z}(t)-\left(\mathbf{F}-\varphi(y, r) \mathbf{A}_{12}^{\mathrm{T}} \mathbf{P}\right) \cdot \mathbf{B}_{2} \cdot \widehat{d}_{2}(t) \\
& \left.+k_{1} s_{n}(\mathbf{z}, t)+\widehat{k}(t) \cdot \operatorname{sgn}\left(s_{n}(\mathbf{z}, t)\right)\right)
\end{aligned}
$$

forces the trajectory error of system described by (6) to move from any initial condition to the sliding surface (33) in finite time and thereafter to remain on it, where $k_{1}>0$.

Proof. Let a Lyapunov function for system (6) be given as

$$
V_{3}(t)=\frac{1}{2} s_{n}^{2}(z, t)+\frac{1}{2 \mu}\left(\widehat{k}(t)-q_{3}\right)^{2} .
$$

Using the controller of (36) and (33), it follows that

$$
\begin{aligned}
\dot{V}_{3}(t)=s_{n}(\mathbf{z}, t) \cdot \dot{s}_{n}(\mathbf{z}, t)+\frac{1}{\mu}\left(\widehat{k}(t)-q_{3}\right) \dot{\hat{k}}(t) \\
=\frac{1}{\mu}\left(\widehat{k}(t)-q_{3}\right) \dot{\hat{k}}(t)+s_{n}(\mathbf{z}, t) \cdot\left(-k_{1} s_{n}(\mathbf{z}, t)\right. \\
-\widehat{k}(t) \operatorname{sgn}\left(s_{n}(\mathbf{z}, t)\right)-\left(\mathbf{F}-\varphi(y, r) \mathbf{A}_{12}^{\mathrm{T}}\right) \mathbf{P} \cdot \mathbf{B}_{2} \\
\cdot d(t)-B_{1} \cdot d_{1}(t)+\left(\mathbf{F}-\varphi(y, r) \mathbf{A}_{12}^{\mathrm{T}}\right) \mathbf{P} \cdot \mathbf{B}_{2} \\
\left.\cdot \widehat{d}_{2}(t)+h\left(d_{2}(t)-\widehat{d}_{2}(t)\right)\right) \leq-k_{1} s_{n}^{2}(\mathbf{z}, t)-\left(q_{3}\right. \\
\left.-|\delta(y, r)|-\left|B_{1} \cdot d_{1}(t)\right|\right) \cdot\left|s_{n}(\mathbf{z}, t)\right| .
\end{aligned}
$$

From (35), (38) implies that $\dot{V}_{3}(t)<0$. This completes the proof.

The block diagram of ANSMC-DOB is shown in Figure 7. In Figure 7, the matrixes $\mathbf{H}$ and $\boldsymbol{\Gamma}$ are, respectively, represented as

$$
\begin{aligned}
\mathbf{H} & =\left[\begin{array}{llll}
0 & 0 & h & 0
\end{array}\right] \\
\boldsymbol{\Gamma} & =\left[\begin{array}{llll}
\frac{h k}{m_{2}} & -\frac{h k}{m_{2}} & -h^{2}+h \frac{b_{2}+c}{m_{2}}-\frac{h c}{m_{2}}
\end{array}\right]
\end{aligned}
$$

To prevent the adaptive parameter estimation from increasing infinitely, the following equation with a small threshold value $\varepsilon$ is used to improve (21):

$$
\dot{\hat{k}}(t)= \begin{cases}\mu|s(\mathbf{z}, t)|, & |s(\mathbf{z}, t)| \geq \varepsilon \\ 0, & \text { else. }\end{cases}
$$

Meanwhile, in order to weaken the chattering brought about by the switching control in ANSMC-DOB, a saturation function as follows with a certain threshold value is used here instead of the sign function in the control law (36):

$$
\text { sat }(u)= \begin{cases}\operatorname{sgn}(u), & |u|>\ell \\ \frac{1}{\ell} u, & |u| \leq \ell .\end{cases}
$$




\section{Simulation Research and Comparative Experiments}

5.1. Simulation Research. In this section, a set of simulations are conducted to evaluate the tracking performance of pickplace actuation axis with two-DOF model (1a) and (1b) under the condition of matched and mismatched disturbance existing, respectively. The reference trajectory is a jerk continuous signal shown in Figure 8. The maximum acceleration is $8 \mathrm{~m} / \mathrm{s}^{2}$ and the maximum velocity is $1 \mathrm{~m} / \mathrm{s}$. This is equivalent to a back-and-forth motion for the actuator in the pick-place actuation axis of SMT assembly machine. Two adaptive sliding mode controllers based on linear sliding surface (ALSMC) with different damping ratios and ANSMC are used in the simulation research. The damping ratios of ALSMC-1 and ALSMC-2 are 0.5 and 0.99, respectively. These values are the initial and ultimate damping ratio in succession for ANSMC controller. The ALSMC control law can be acquired from (23) under the circumstance of $\varphi(y, r)=$ 0 . The system parameter perturbations and disturbances in simulations are hypothesized as follows:

$$
\begin{aligned}
& d_{1}(t)=10 \sin (50 t), \\
& d_{2}(t)=10 \sin (100 t) .
\end{aligned}
$$

Figure 9 shows the tracking errors and control signals for the three controllers without the mismatched disturbance. It can be seen that the proposed ANSMC achieves the minimum tracking error and is more robust to the matched disturbance compared to ALSMC.

Figure 10 gives the superiority of ANSMC-DOB over ANSMC in dealing with the mismatched disturbance of the system. From Figure 10, it can be seen that the ANSMCDOB controller proposed in this paper can reduce the corresponding tracking error effectively. Figure 11 shows the comparison of $d_{2}(t)$ and $\widehat{d}_{2}(t)$. It is obviously seen that the mismatched disturbance can be accurately estimated by the proposed disturbance observer.

5.2. Comparative Experiments. In order to highlight the effectiveness of the proposed ANSMC-DOB, the comparative control experiments are implemented for the pick-place actuation axis of machine in dSPACE DS1104 system with the reference trajectory shown in Figure 8. With this trajectory, the actuator is firstly moving to the left side and then back to the initial location. The following four controllers are compared and the sampling time of controllers implemented in DS1104 is $16 \mathrm{kHz}$.

(1) PID. Well-tuned PID is used for the linear position feedback control loop. Classical PID is adopted which is designed as follows:

$$
\begin{aligned}
& e_{x 2}(t)=x_{2 r}(t)-x_{2}(t), \\
& u_{1}(t)=K_{p}\left(e_{x 2}(t)+\frac{1}{T_{i}} \int e_{x 2}(t) \mathrm{d} t+T_{d} \frac{\mathrm{d} e_{x 2}(t)}{\mathrm{d} t}\right),
\end{aligned}
$$

where $K_{p}, T_{i}$, and $T_{d}$ are the proportion coefficient, integration time constant, and derivative time constant, respectively.
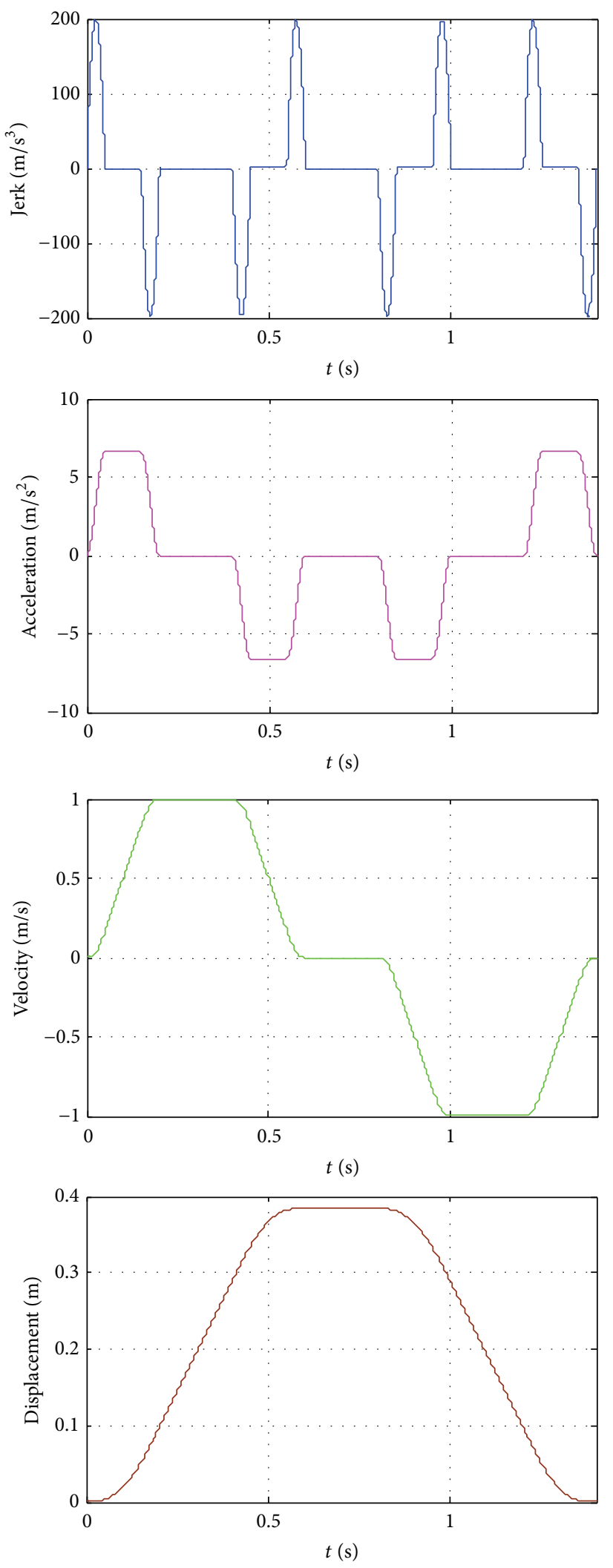

FiguRE 8: Reference trajectory.

In order to avoid high frequency interference caused by the derivative action, a low pass filter is introduced. Figure 12 

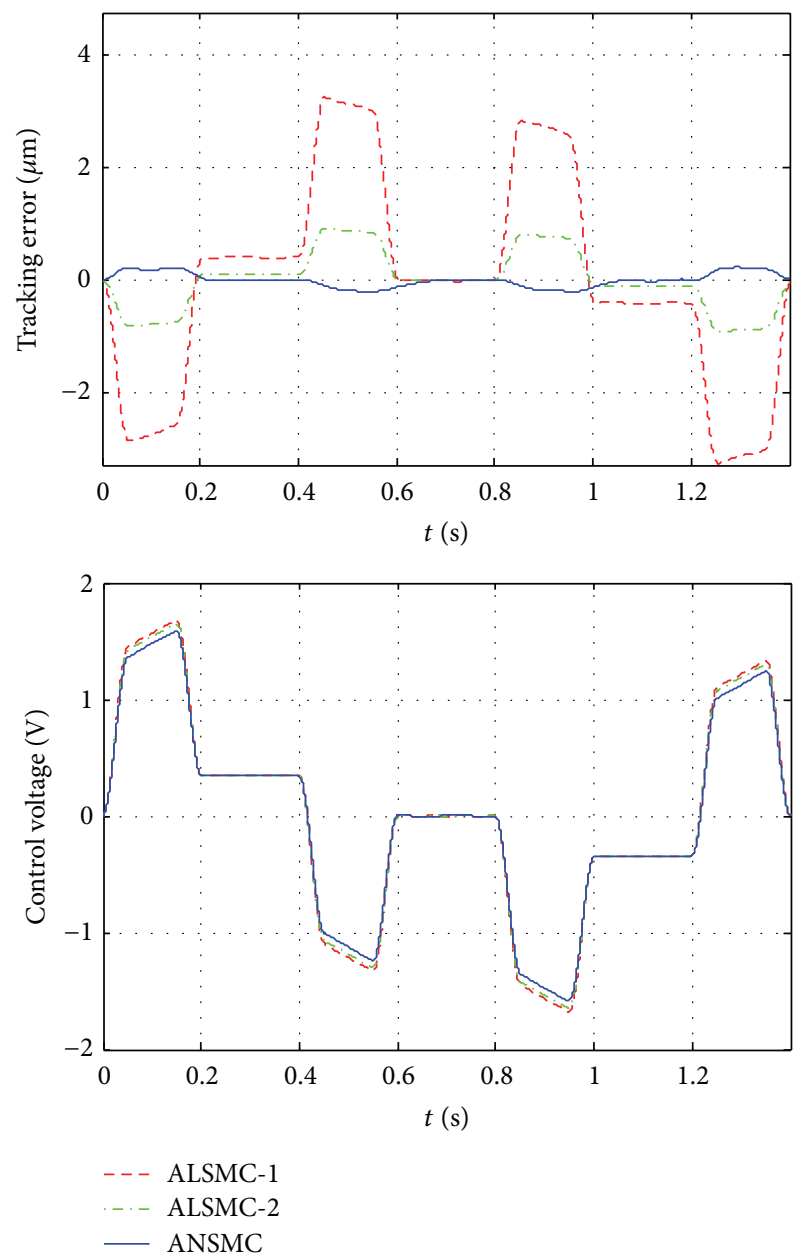

FIgURE 9: Three control strategies' simulation comparison.

shows the block diagram of PID controller. The saturation part is used for limiting the control signal in case of the outof-range voltage.

(2) ALSMC. The ALSMC control law can be acquired from (23) under the circumstance of $\varphi(y, r)=0$. The coefficient matrix of linear sliding surface is chosen as $\mathbf{c}=\left[\begin{array}{ll}\mathbf{F} & 1\end{array}\right]=$ $\left[\begin{array}{llll}12752.39 & 8346.9 & 33.3184 & 1\end{array}\right]$ to make the closed-loop poles (i.e., eigenvalues of $\left[\mathbf{A}_{11}-\mathbf{A}_{12} \mathbf{F}\right]$ ) as -7500 and $-764.4123 \pm$ j633.4138. The damping ratio of the dominant poles is 0.77 .

(3) ANSMC. In order to satisfy the initial dominant pole condition of small damping ratio and stability performance, the matrix $\mathbf{F}$ is calculated as $\mathbf{F}=\left[\begin{array}{lll}8395.3 & 5670.9 & 15.1650\end{array}\right]$. The nonlinear function used in the nonlinear sliding surface is chosen as

$$
\varphi(y, r)=-3 \cdot e^{-5(y-r)^{2}} .
$$

When the actuator is moving close to the reference trajectory, $\varphi(y, r)$ is approaching -3 . Afterwards, the eigenvalues of state error subsystem $\mathbf{e}_{1}(t)$ are calculated as -4.837 and $-31656.72 \pm j 4014.22$. The corresponding damping ratios are

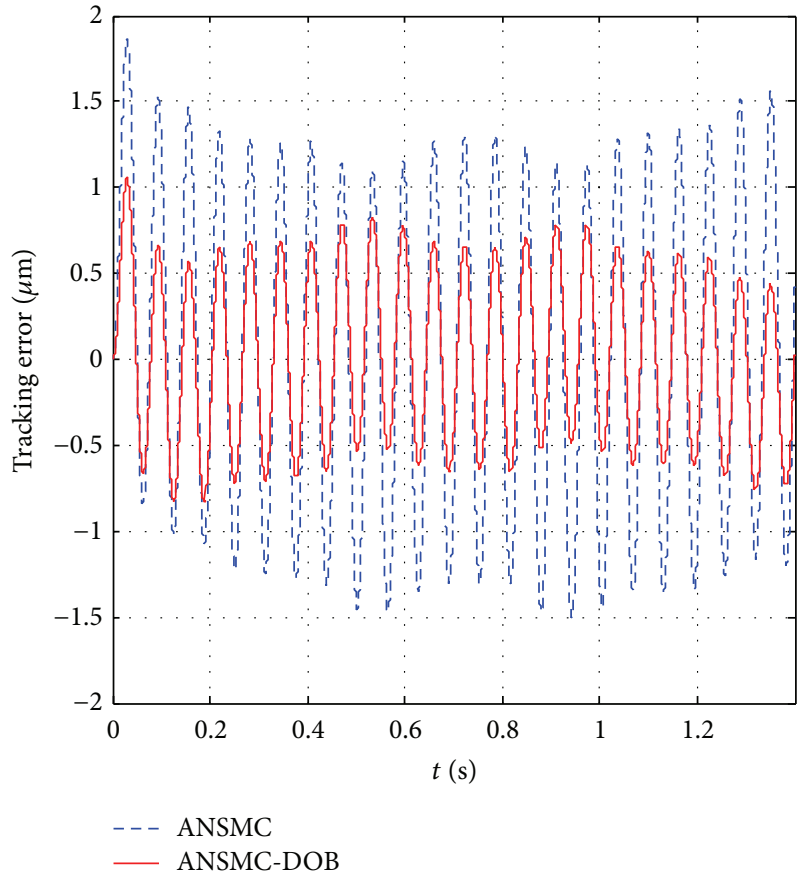

FIgUre 10: The comparison between ANSMC and ANSMC-DOB when $d_{1}(t)=10 \sin (50 t)$ and $d_{2}(t)=10 \sin (100 t)$.

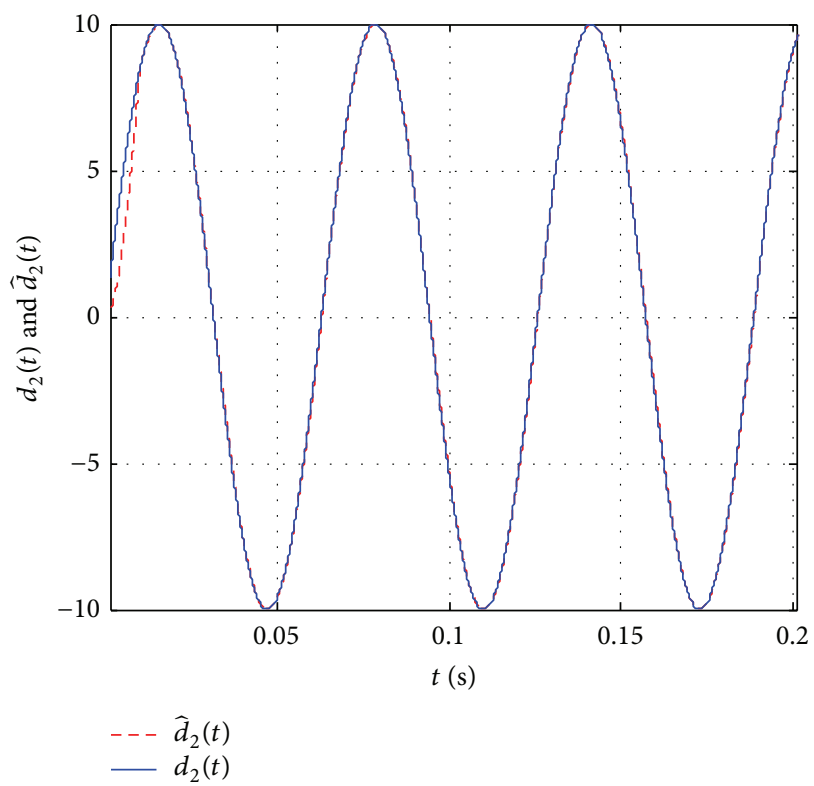

FIGURE 11: Comparison of $d_{2}(t)$ and $\widehat{d}_{2}(t)$.

1.0, 0.992 , and 0.992 which satisfy the big damping ratio requirements.

(4) ANSMC-DOB. The disturbance observer is integrated into ANSMC described above to generate the ANSMC-DOB controller (36). The parameter $h$ of disturbance observer (28a) and (28b) is chosen as $h=1$. 


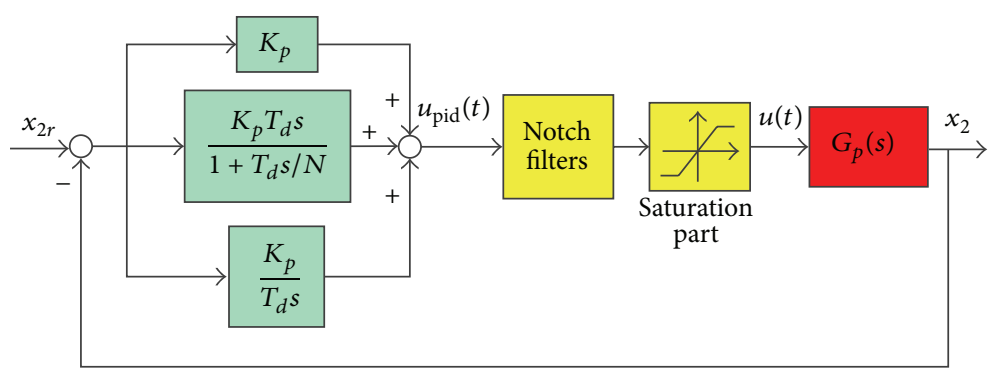

FIGURE 12: The block diagram of PID controller.
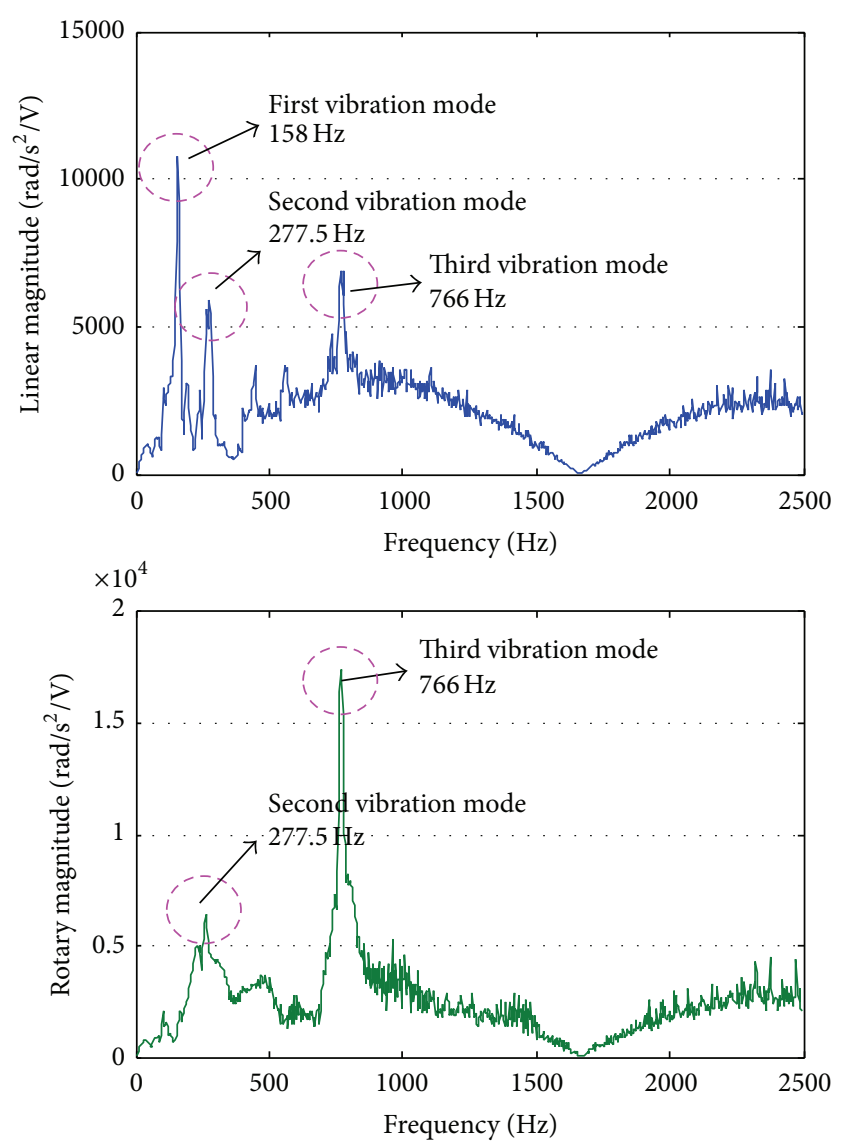

FIGURE 13: Frequency response measured from rotary and grating ruler encoders.

The notch filters are designed according to the frequency response experiment to suppress the high-order vibrations of the pick-place actuation axis. The operation of frequency response experiment is to give the servo motor a sinusoidal input voltage as torque command. The frequencies of the torque command signal are ranging from $1 \mathrm{~Hz}$ to $2500 \mathrm{~Hz}$. Figure 13 shows the frequency response result which is, respectively, measured from grating ruler encoder and rotary encoder. It can be seen from Figure 13 that, apart from the first axial vibration, the other measuring dominant vibration modes of the pick-place actuation axis are, respectively, $277.5 \mathrm{~Hz}$ and $766 \mathrm{~Hz}$. The second and third vibration modes are the strong coupling between the axial and torsional vibration of ball screw. The corresponding notch filters are designed as follows:

$$
\begin{aligned}
& N_{1}(z)=\frac{1-1.985 z^{-1}+0.9947 z^{-2}}{1-1.781 z^{-1}+0.7932 z^{-2}}, \\
& N_{2}(z)=\frac{1-1.894 z^{-1}+0.9769 z^{-2}}{1-1.459 z^{-1}+0.5442 z^{-2}} .
\end{aligned}
$$

In the control system of pick-place actuation axis, there is strong nonlinear behavior including parameter perturbations and external disturbance. The parameter perturbation always exists during the process of actuator moving as described before. The external disturbance mostly consists of the load disturbance which is the actions of 8 nozzles in the actuator. During the process of the actuator moving, there is only the rotating motion of nozzles driven by eight small servo motors. The vertical motion of the nozzles is executed when the actuator stops moving to avoid the collision. When the actuator moves as the reference trajectory of Figure 8, the 8 nozzles are driven simultaneously to move rotationally at $t=0.3 \mathrm{~s}$. Figure 14 shows the comparative results of the above four controllers when the actuator is in the middle mark of ball screw initially. From Figure 14, it can be seen that the positioning accuracy has been decreased by ALSMC and ANSMC in the whole movement process compared with PID. There is an obvious increase point of tracking error controlled by PID at $t=0.3 \mathrm{~s}$ while the tracking errors controlled by ALSMC and ANSMC have no distinct changes. It embodies that the ball screw driven system of SMT assembly machine controlled by ALSMC and ANSMC not only reduces the tracking error when the time-varying parameter uncertainties exist, but also has the robustness to the external disturbance. Due to the gentle variations of damping ratio in ANSMC, it can be seen from Figure 14 that the use of ANSMC in the control system avoids the big control voltage jump while in contrast with ALSMC. Meanwhile, the tracking error of the actuator is reduced by around $10 \sim 15 \mu \mathrm{m}$ by the proposed ANSMC-DOB compared to PID, ALSMC, and ANSMC controllers. The positioning error range of actuator by ANSMC-DOB is $\pm 10 \mu \mathrm{m}$ which satisfies the design requirements. 

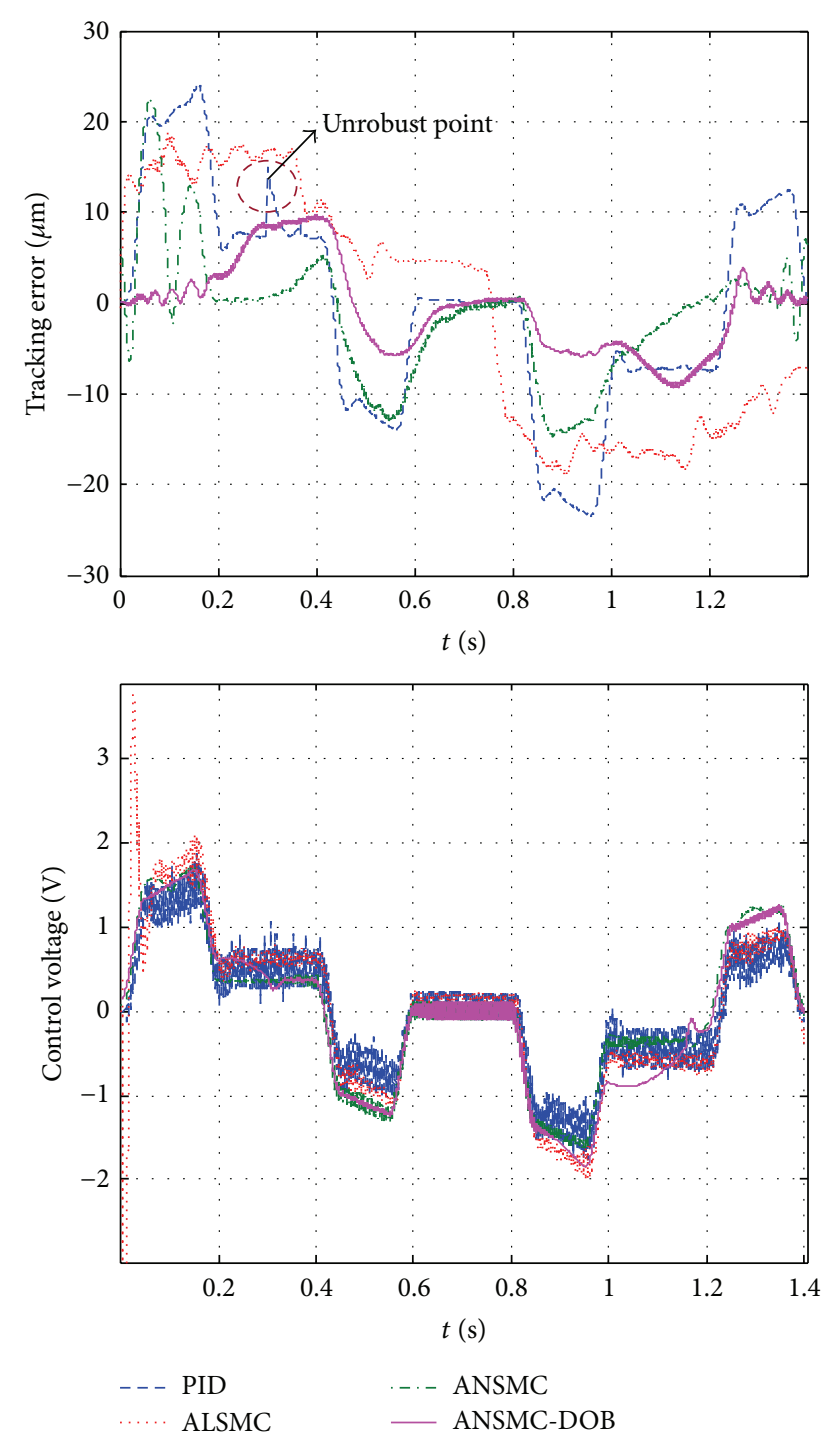

FIGURE 14: Comparative results of four controllers.

For the long travel of ball screw system, the high-order mode frequency of dynamic performance shifts for different positions of the actuator $[38,39]$. This means that the influence of notch filters may decrease and the uncompleted dynamic model and uncertainty increased. In order to verify the robustness of the proposed ANSMC-DOB, the other two experiments are conducted by making the actuator locate initially at the extreme positions which are the left and right marks. Figure 15 shows the tracking errors of these two conditions. It can be seen that the tracking error of the actuator is still controlled in the range of $\pm 10 \mu \mathrm{m}$. The partial variation of dynamic performance is considered as the disturbance which is estimated by the disturbance observer. From Figures 14 and 15, it can be concluded that the proposed ANSMC-DOB is more robust to the uncompleted dynamic model uncertainty and the external disturbance to acquire more precision position accuracy.

The procedure of parameter estimation $\widehat{k}(t)$ for three different initial positions of actuator is shown in Figure 16. It can

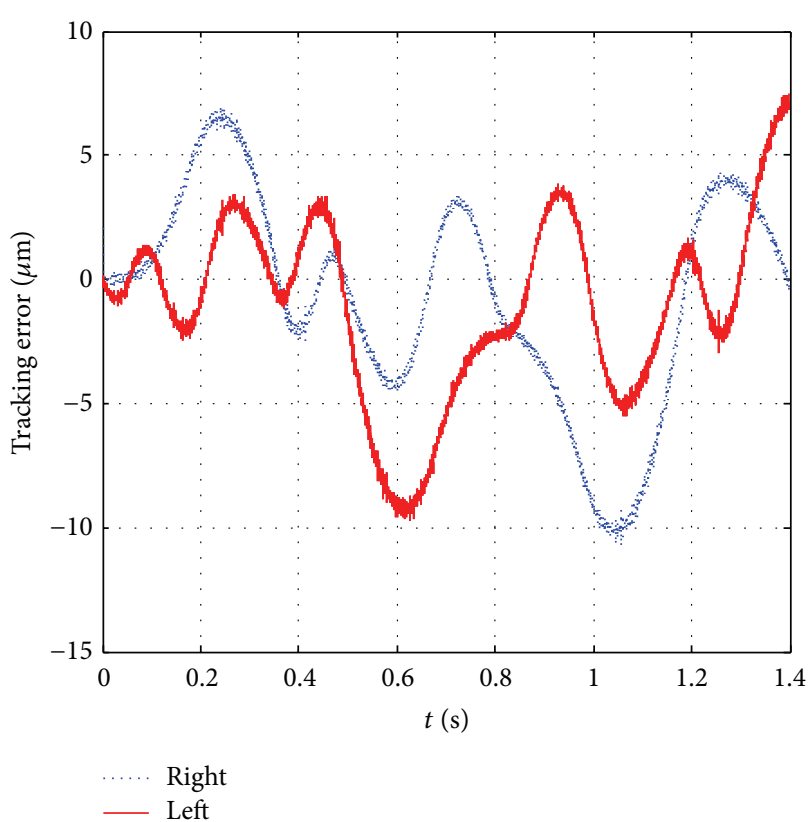

FiguRE 15: Tracking errors at two extreme positions.

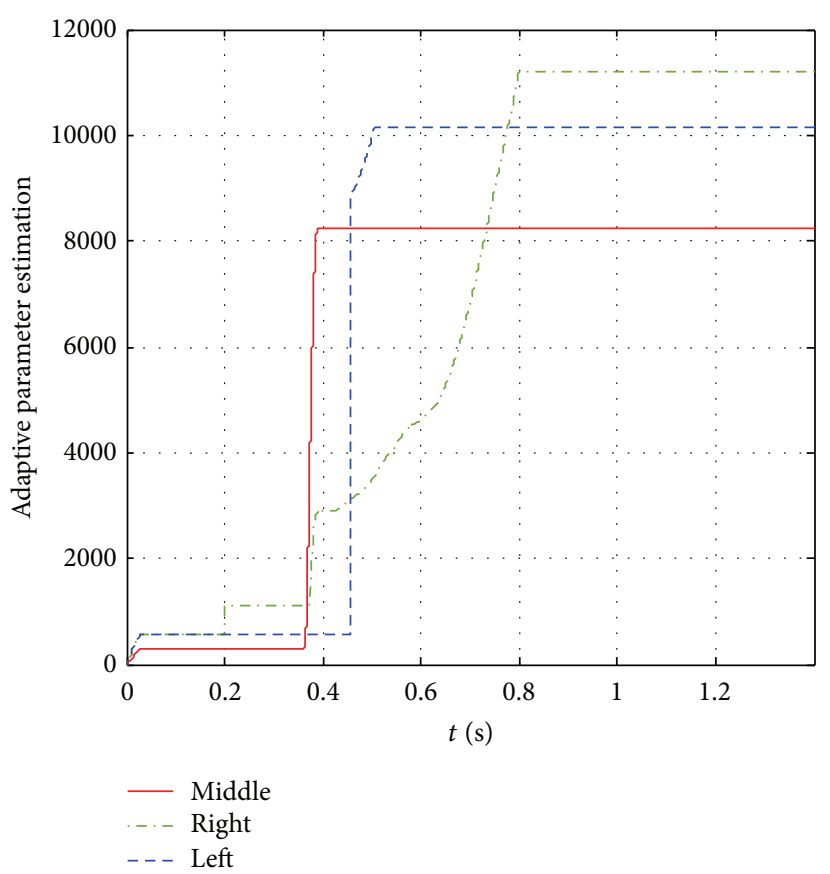

FIGURE 16: The procedure of parameter estimation $\widehat{k}(t)$.

be seen that $\widehat{k}(t)$ always keeps unchanged eventually when the sliding surface remains in the threshold. The threshold here is used for avoiding huge chattering caused by the infinite growth of $\widehat{k}(t)$. Figure 17 shows the observation result by disturbance observer (28a) and (28b) under different initial positions of the actuator. The disturbance always combines the friction characteristics and the dynamic variation caused by the actuator moving. 


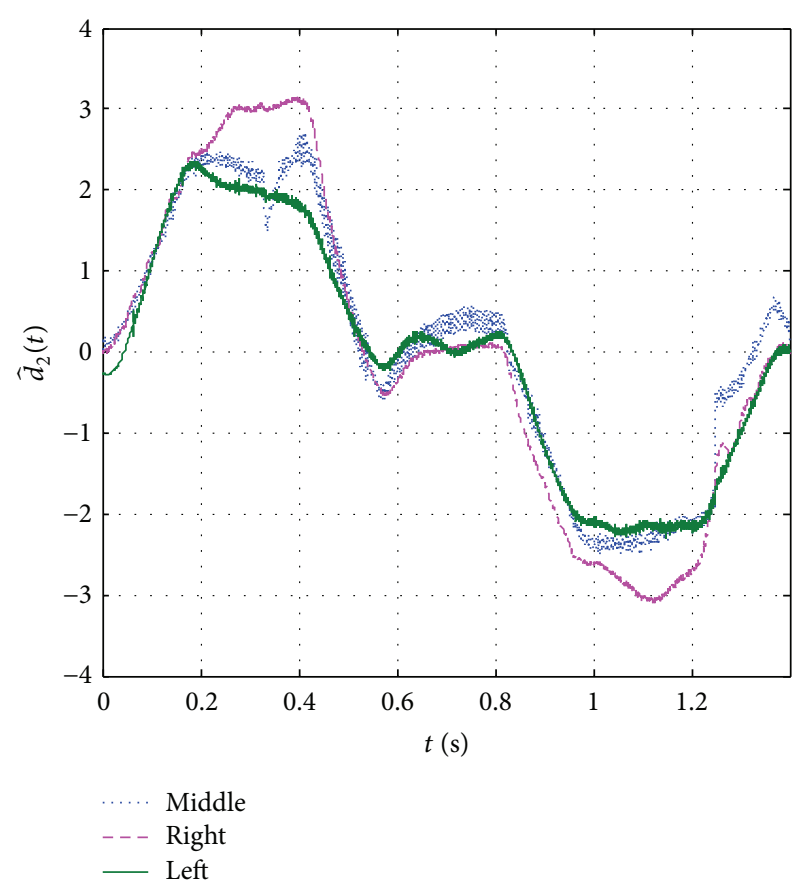

FIGURE 17: The observation result of $\hat{d}_{2}(t)$.

\section{Conclusion}

An ANSMC-DOB controller has been proposed for the SMT assembly machine to achieve fast and precision trajectory tracking control. Combining the merits of nonlinear sliding surface and disturbance observer, the proposed method has the advantages of good transient performance and robustness to both the matched and the mismatched disturbance. The results of simulation research and comparative experiments indicate that the proposed ANSMC-DOB can achieve fast and precision positioning performance for SMT assembly machine. This control strategy provides a new solution for high performance tracking control applications. In the future, the friction characteristic should be modeled accurately and the feed-forward controller can be designed combining with ANSMC-DOB to alleviate the friction to obtain more accurate tracking.

\section{Competing Interests}

The authors declare that they have no competing interests.

\section{Acknowledgments}

This paper is supported by the National Science-Technology Support Plan Projects of China (Grant no. 2015BAK06B02) and the National Natural Science Foundation of China (Grant no. 51405470).

\section{References}

[1] P. H. Chang and J. Park, "A concurrent design of input shaping technique and a robust control for high-speed/high-precision control of a chip mounter," Control Engineering Practice, vol. 9, no. 12 , pp. 1279-1285, 2001.

[2] H. Ding and J. Wu, "Point-to-point motion control for a highacceleration positioning table via cascaded learning schemes," IEEE Transactions on Industrial Electronics, vol. 54, no. 5, pp. 2735-2744, 2007.

[3] K. Erkorkmaz and Y. Altintas, "High speed CNC system design. Part I. Jerk limited trajectory generation and quintic spline interpolation," International Journal of Machine Tools and Manufacture, vol. 41, no. 9, pp. 1323-1345, 2001.

[4] H. Li, M. D. Le, Z. M. Gong, and W. Lin, "Motion profile design to reduce residual vibration of high-speed positioning stages," IEEE/ASME Transactions on Mechatronics, vol. 14, no. 2, pp. 264-269, 2009.

[5] X. Shi and S. Chang, "Extended state observer-based time optimal control for fast and precision point-to-point motions driven by a novel electromagnetic linear actuator," Mechatronics, vol. 4, no. 4, pp. 445-451, 2013.

[6] D. J. Gordon and K. Erkorkmaz, "Accurate control of ball screw drives using pole-placement vibration damping and a novel trajectory prefilter," Precision Engineering, vol. 37, no. 2, pp. 308322, 2013

[7] Y. Hosseinkhani and K. Erkorkmaz, "High frequency harmonic cancellation in ball-screw drives," Procedia CIRP, vol. 1, no. 7, pp. 615-620, 2012.

[8] H. Zhang and F. L. Lewis, "Adaptive cooperative tracking control of higher-order nonlinear systems with unknown dynamics," Automatica, vol. 48, no. 7, pp. 1432-1439, 2012.

[9] B. Xiao, Q. Hu, and Y. Zhang, "Adaptive sliding mode fault tolerant attitude tracking control for flexible spacecraft under actuator saturation," IEEE Transactions on Control Systems Technology, vol. 20, no. 6, pp. 1605-1612, 2012.

[10] J. Yao, Z. Jiao, D. Ma, and L. Yan, "High-accuracy tracking control of hydraulic rotary actuators with modeling uncertainties," IEEE/ASME Transactions on Mechatronics, vol. 19, no. 2, pp. 633-641, 2014.

[11] B. Jia, S. Liu, and L. Liu, "Visual trajectory tracking of industrial manipulator with iterative learning control," Industrial Robot, vol. 42, no. 1, pp. 54-63, 2015.

[12] H. Chen and S. Wo, "RBF neural network of sliding mode control for time-varying 2-DOF parallel manipulator system," Mathematical Problems in Engineering, vol. 2013, Article ID 201712, 10 pages, 2013.

[13] C. L. Chen, M. J. Jang, and K. C. Lin, "Modeling and highprecision control of a ball-screw-driven stage," Precision Engineering, vol. 28, no. 4, pp. 483-495, 2004.

[14] K. Erkorkmaz and A. Kamalzadeh, "High bandwidth control of ball screw drives," CIRP Annals-Manufacturing Technology, vol. 55, no. 1, pp. 393-398, 2006.

[15] L. Dong and W. C. Tang, "Adaptive backstepping sliding mode control of flexible ball screw drives with time-varying parametric uncertainties and disturbances," ISA Transactions, vol. 53, no. 1, pp. 110-116, 2014.

[16] B. Yao and M. Tomizuka, "Smooth robust adaptive sliding mode control of manipulators with guaranteed transient performance," in Proceedings of the American Control Conference (ACC '94), vol. 1, pp. 1176-1180, IEEE, Baltimore, Md, USA, June-July 1994.

[17] L. Xu and B. Yao, "Adaptive robust precision motion control of linear motors with negligible electrical dynamics: theory and experiments," IEEE/ASME Transactions on Mechatronics, vol. 6, no. 4, pp. 444-452, 2001. 
[18] J. Zhong and B. Yao, "Adaptive robust precision motion control of a piezoelectric positioning stage," IEEE Transactions on Control Systems Technology, vol. 16, no. 5, pp. 1039-1046, 2008.

[19] C. P. Bechlioulis and G. A. Rovithakis, "Robust adaptive control of feedback linearizable MIMO nonlinear systems with prescribed performance," IEEE Transactions on Automatic Control, vol. 53, no. 9, pp. 2090-2099, 2008.

[20] C. P. Bechlioulis and G. A. Rovithakis, "A priori guaranteed evolution within the neural network approximation set and robustness expansion via prescribed performance control," IEEE Transactions on Neural Networks and Learning Systems, vol. 23, no. 4, pp. 669-675, 2012.

[21] Z. Doulgeri and L. Droukas, "On rolling contact motion by robotic fingers via prescribed performance control," in Proceedings of the IEEE International Conference on Robotics and Automation (ICRA '13), pp. 3976-3981, IEEE, Karlsruhe, Germany, May 2013.

[22] B. Bijnan, D. Fulwani, and L. Fridman, Sliding Mode Control Using Novel Sliding Surface, Springer, London, UK, 2009.

[23] Z. Lin, M. Pachter, and S. Banda, "Toward improvement of tracking performance-nonlinear feedback for linear systems," International Journal of Control, vol. 70, no. 1, pp. 1-11, 1998.

[24] D. Ginoya, P. D. Shendge, and S. B. Phadke, "Sliding mode control for mismatched uncertain systems using an extended disturbance observer," IEEE Transactions on Industrial Electronics, vol. 61, no. 4, pp. 1983-1992, 2014.

[25] F. Zhao, Y. Liu, X. Yao, and B. Su, "Integral sliding mode control of time-delay systems with mismatching uncertainties," Journal of Systems Engineering and Electronics, vol. 21, no. 2, pp. 273280, 2010.

[26] V. Q. Leu, H. H. Choi, and J.-W. Jung, "LMI-based sliding mode speed tracking control design for surface-mounted permanent magnet synchronous motors," Journal of Electrical Engineering \& Technology, vol. 7, no. 4, pp. 513-523, 2012.

[27] J. Zhang, P. Shi, and Y. Xia, "Robust adaptive sliding-mode control for fuzzy systems with mismatched uncertainties," IEEE Transactions on Fuzzy Systems, vol. 18, no. 4, pp. 700-711, 2010.

[28] S. W. Wang, D. W. Yu, and D. L. Yu, "Compensation for unmatched uncertainty with adaptive RBF network," International Journal of Engineering, Science and Technology, vol. 3, no. 6, pp. 35-43, 2012.

[29] K.-S. Kim, Y. Park, and S.-H. Oh, "Designing robust sliding hyperplanes for parametric uncertain systems: a Riccati approach," Automatica, vol. 36, no. 7, pp. 1041-1048, 2000.

[30] M. Chen and W.-H. Chen, "Sliding mode control for a class of uncertain nonlinear system based on disturbance observer," International Journal of Adaptive Control and Signal Processing, vol. 24, no. 1, pp. 51-64, 2010.

[31] X. Wei and L. Guo, "Composite disturbance-observer-based control and terminal sliding mode control for non-linear systems with disturbances," International Journal of Control, vol. 82, no. 6, pp. 1082-1098, 2009.

[32] Y.-S. Lu and C.-W. Chiu, "A stability-guaranteed integral sliding disturbance observer for systems suffering from disturbances with bounded first time derivatives," International Journal of Control, Automation and Systems, vol. 9, no. 2, pp. 402-409, 2011.

[33] X. Chen, S. Komada, and T. Fukuda, "Design of a nonlinear disturbance observer," IEEE Transactions on Industrial Electronics, vol. 47, no. 2, pp. 429-437, 2000.
[34] X.-W. Bu, X.-Y. Wu, Y.-X. Chen, and R.-Y. Bai, "Design of a class of new nonlinear disturbance observers based on tracking differentiators for uncertain dynamic systems," International Journal of Control, Automation and Systems, vol. 13, no. 3, pp. 595602, 2015.

[35] A. Kamalzadeh, Precision Control of High Speed Ball Screw Drives, University of Waterloo, Waterloo, Canada, 2008.

[36] W.-H. Chen, "Nonlinear disturbance observer-enhanced dynamic inversion control of missiles," Journal of Guidance, Control, and Dynamics, vol. 26, no. 1, pp. 161-166, 2003.

[37] J. Yang, S. Li, and X. Yu, "Sliding-mode control for systems with mismatched uncertainties via a disturbance observer," IEEE Transactions on Industrial Electronics, vol. 60, no. 1, pp. 160-169, 2013.

[38] D. A. Vicente, R. L. Hecker, F. J. Villegas, and G. M. Flores, "Modeling and vibration mode analysis of a ball screw drive," International Journal of Advanced Manufacturing Technology, vol. 58, no. 1-4, pp. 257-265, 2012.

[39] H. Zhu and H. Fujimoto, "Mechanical deformation analysis and high-precision control for ball-screw-driven stages," IEEE/ ASME Transactions on Mechatronics, vol. 20, no. 2, pp. 956-966, 2015. 


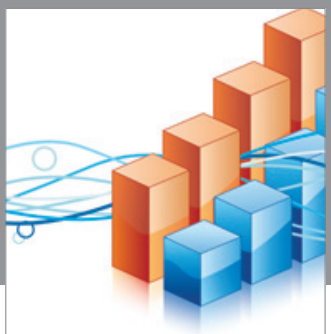

Advances in

Operations Research

vatem alat4

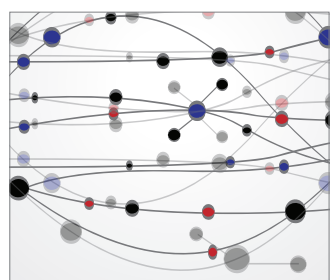

\section{The Scientific} World Journal
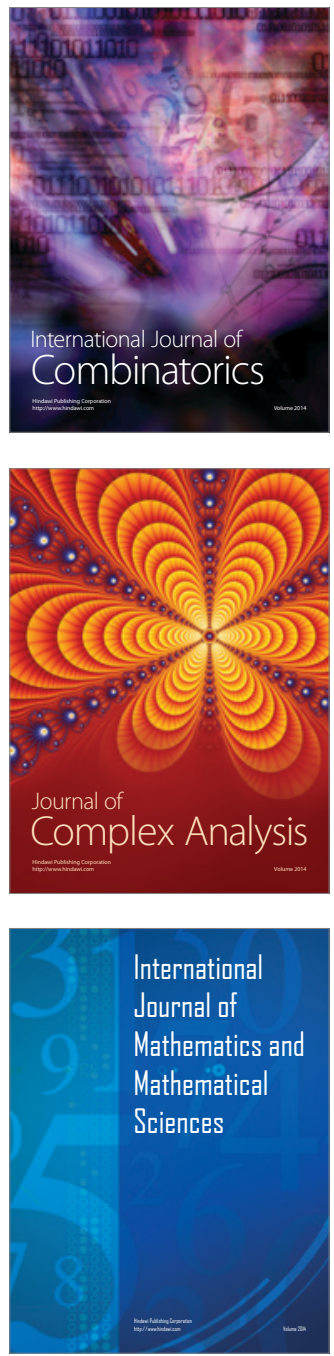
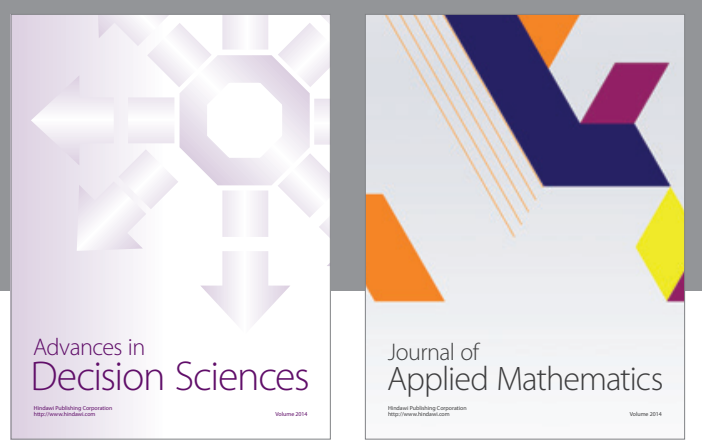

Algebra

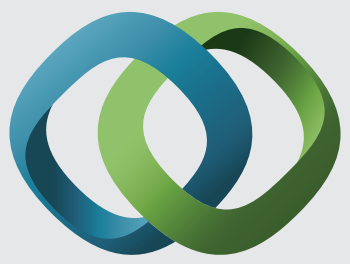

\section{Hindawi}

Submit your manuscripts at

http://www.hindawi.com
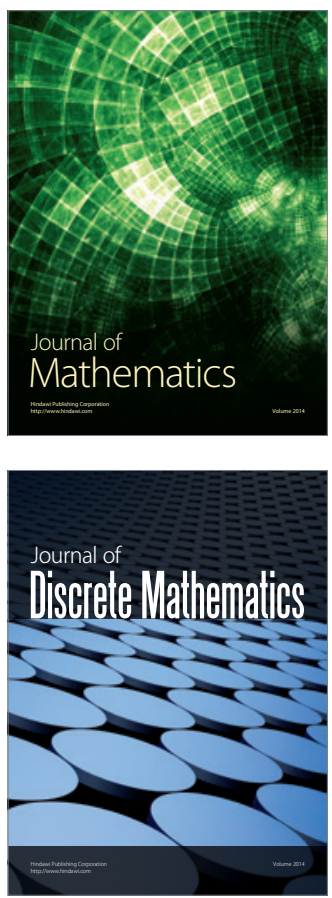

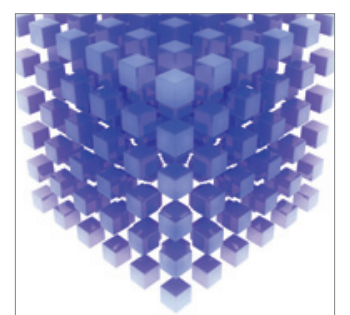

Mathematical Problems in Engineering
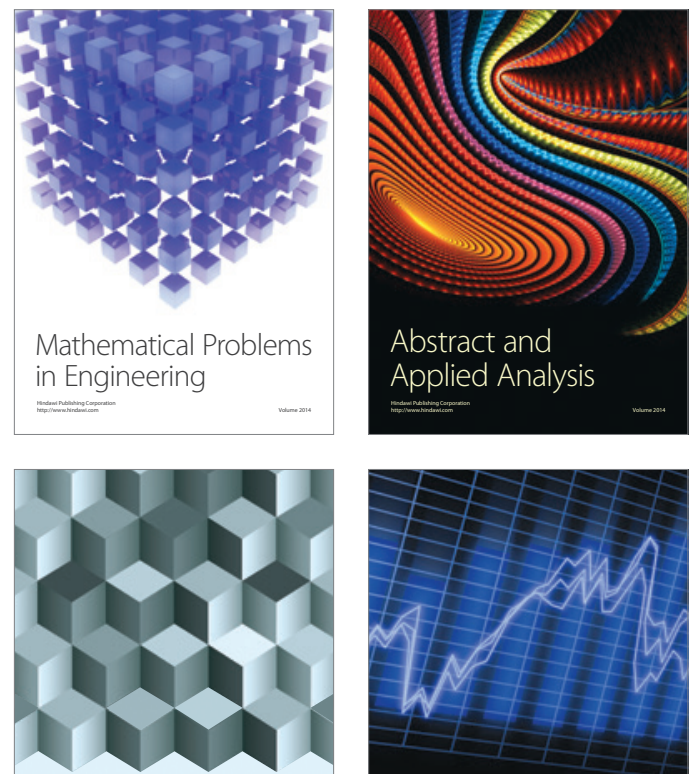

Journal of

Function Spaces

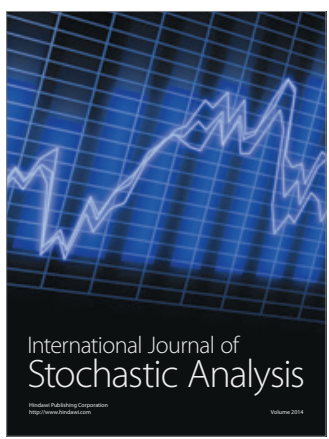

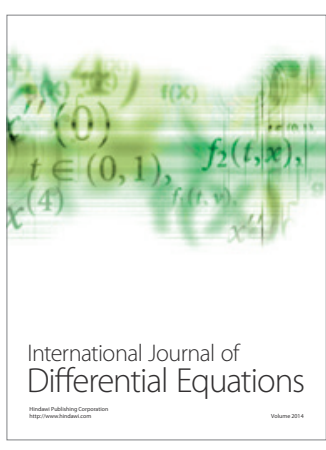
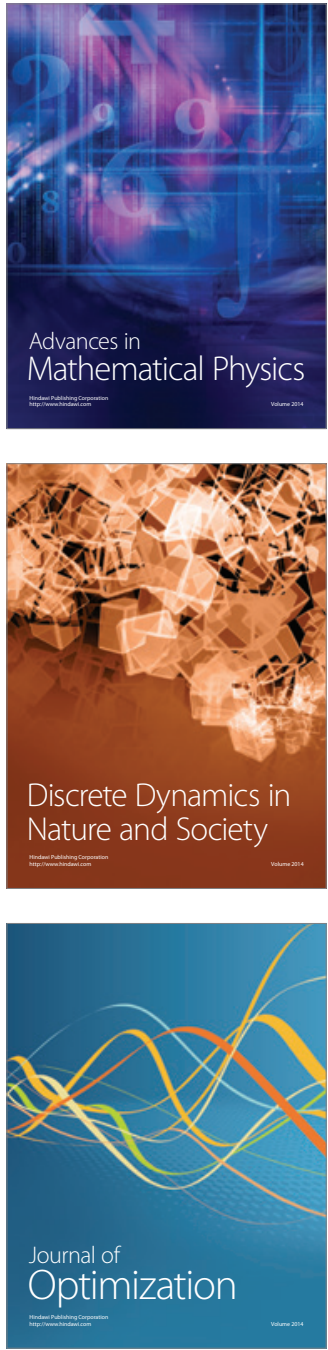